\title{
El potencial de OneNote para el desarrollo de la competencia digital docente
}

\author{
Manuela Mena Octavio \\ Directora académica de The Language House/Fundadora de feelthelanguage.com/ \\ Profesora en la Universidad Nebrija (España) y en SDI Múnich (Alemania) \\ mmena30@gmail.com | https://orcid.org/0000-0002-6937-2959 \\ María Vicenta González Argüello \\ Profesora de la Facultad de Educación de la Universidad de Barcelona (España) \\ vicentagonzalez@ub.edu | https://orcid.org/0000-0002-5262-9500
}

\section{Extracto}

La situación de pandemia generada por la COVID-19 ha puesto en jaque a los diferentes agentes de la comunidad educativa, obligándoles a realizar una transición forzosa a modelos de aula cada vez más digitalizados y tecnológicos. Esta precipitada migración hacia un nuevo paradigma educativo ha supuesto un aumento de la carga de trabajo para los docentes, quienes han tenido que reestructurar y repensar los contenidos y materiales didácticos hasta ahora empleados en el modelo de aula tradicional. Asimismo, el desarrollo de la competencia digital docente (CDD) se ha convertido en el principal objetivo de las instituciones educativas con el fin de satisfacer las nuevas demandas del proceso de enseñanza-aprendizaje y limitar, a su vez, las desigualdades entre el alumnado derivadas de la carencia de una CDD efectiva. En este contexto se presenta el Cuaderno Digital Colaborativo de Observación (CDCO), una propuesta de plan de observación y formación interno desarrollada en OneNote con el objetivo de favorecer la observación entre iguales y desarrollar la competencia digital docente en contexto.

Palabras clave: desarrollo profesional; competencia digital docente (CDD); DigCompEdu; modelo TPACK; observación; investigación-acción; Microsoft OneNote. 


\title{
OneNote class notebook for enhanced teacher digital competence
}

\author{
Manuela Mena Octavio \\ María Vicenta González Argüello
}

\begin{abstract}
The pandemic situation generated by COVID-19 has put the different agents of the educational community in check, forcing them to make a forced transition to increasingly digitalised and technological classroom models. This hasty migration to a new educational paradigm has led to an increased workload for teachers, who have had to restructure and rethink the content and teaching materials hitherto used in the traditional classroom model. Furthermore, the development of teachers' digital competence (TDC) has become the main objective for educational institutions in order to meet the new demands of the learning process and to limit, in turn, the inequalities among students resulting from the students resulting from a lack of effective TDC. In this context, we present the Collaborative Digital Observation Notebook (CDON), a proposal for an internal observation and training plan developed in OneNote with the aim of promoting peer observation and developing digital competence in teaching in context.
\end{abstract}

Keywords: professional development; Teachers'digital competence (TDC); DigCompEdu; TPACK model; observation; action research; Microsoft OneNote. 


\section{Sumario}

1. Justificación

2. La CDD: definición y documentos de referencia

2.1. La autoevaluación de la CDD

2.2. SELFIE: programa europeo para la competencia digital de la comunidad educativa

2.3. El modelo TPACK como marco para el desarrollo de la competencia digital

3. La observación como herramienta para el desarrollo de la CDD

4. Propuesta de diseño de un plan de información a través de un CDCO

4.1. Destinatarios

4.2. Metodología

4.3. Perfil del profesorado observador

5. Cuaderno Digital Colaborativo de Observación (CDCO)

5.1. Justificación de la selección de la herramienta OneNote

5.2. Objetivos

5.3. Contenido

5.3.1. Manual de usuario

5.3.2. Diagnóstico del centro

5.3.3. Diagnóstico CDD

5.3.4. Planificación

5.3.5. Acción

5.3.6. Observación

5.3.7. Reflexión

5.3.8. Mis herramientas

5.3.9. Ejemplo de caso

6. Evaluación del diseño del CDCO

7. Conclusiones e implicaciones futuras

Referencias bibliográficas 


\section{Justificación}

La situación sanitaria derivada de la COVID-19 ha cambiado radicalmente nuestra manera de interpretar el mundo a nuestro alrededor, nuestros hábitos y rutinas. De igual modo, el ámbito educativo, en general, y el de la formación de profesores, en particular, también se ha visto alterado, pasando de un modelo de formación docente predominantemente presencial a un contexto formativo puramente online. Si bien al principio, docentes y alumnado se manifestaron un poco reticentes a los vertiginosos cambios, poco a poco la comunidad educativa se ha ido adaptando a la nueva realidad e, incluso, ya empiezan a verse ventajas a los cambios que ha traído consigo la imparable digitalización.

La nueva situación, con el consecuente paso de la presencialidad a la virtualidad, ha tenido implicaciones en el desarrollo de la competencia digital de profesores y alumnos, pues todos han tenido que familiarizarse con nuevas plataformas de videoconferencia, herramientas y aplicaciones digitales hasta entonces desconocidas. Además, estos cambios se han producido en todos los niveles de la enseñanza (educación primaria, secundaria, universitaria, enseñanza de idiomas, formación continua, etc.).

En este sentido, datos como los que maneja el United Nations International Children's Emergency Fund (UNICEF) ${ }^{1}$ justifican la necesidad de desarrollar proyectos que incidan positivamente en el desarrollo de la CDD para la formación continua del profesorado, a saber:

- Casi el $60 \%$ de los miembros de la comunidad educativa encuestados admitió no haber utilizado el aprendizaje a distancia o en línea antes de la llegada de la COVID-19.

- El $95 \%$ de los encuestados considera que la crisis marca un punto de inflexión en cómo se utiliza la tecnología en la educación y la formación.

- Más del 60 \% sintió que habían mejorado sus habilidades digitales durante la crisis.

- En general, la mayor parte de los encuestados está de acuerdo en que los recursos y el contenido de aprendizaje en línea deben ser más relevantes, interactivos e intuitivos.

1 https://www.unicef.org/es/comunicados-prensa/falta-igualdad-acceso-educacion-distancia-podriaagravar-crisis-aprendizaje 
La pandemia ha puesto de relieve muchas deficiencias del sistema educativo y ha agudizado las desigualdades. De acuerdo con una encuesta ${ }^{2}$ realizada por la United Nations Educational, Scientific and Cultural Organization (UNESCO), UNICEF y el Banco Mundial y, posteriormente, puesto de manifiesto por el Observatorio de Innovación Educativa del Tecnológico de Monterrey, solo la mitad de los países que participaron en la encuesta proporcionaron a sus profesores la formación necesaria para poder abordar el reto de la enseñanza a distancia. Por otro lado, y según esa misma encuesta, menos de un tercio de los países ofrecieron ayuda psicológica a sus docentes para que pudieran hacer frente a la situación.

En el mismo informe, se pone de manifiesto que el $81 \%$ de los profesores de primaria de todo el mundo y el $78 \%$ de los docentes de secundaria apenas cumplen con los requisitos para llevar a cabo sus tareas docentes diarias, lo cual demuestra que muchos de estos profesionales no están debidamente preparados para afrontar los problemas que surgen de la nueva situación educativa producida por la pandemia. El caso de Latinoamérica es incluso más preocupante, pues el $83 \%$ de los profesores de primaria y el $84 \%$ de los docentes de secundaria no cuentan con las herramientas para hacer frente a los nuevos retos educativos.

No obstante, no todo es negativo. Una de las ventajas que la situación de pandemia ha traído consigo es que puede afirmarse que la tecnología, en el ámbito de la enseñanza, ha entrado de lleno en la vida del profesorado, lo que ha permitido un rápido desarrollo de su competencia digital gracias al descubrimiento de nuevas herramientas y al desarrollo de habilidades para su óptima integración en la enseñanza. Sin embargo, esta nueva situación también ha evidenciado que los esfuerzos realizados por los docentes son insuficientes y que necesitan seguir formándose para ofrecer respuestas de calidad en sus contextos de trabajo y disminuir con ello las posibles desigualdades educativas entre su alumnado. De hecho, en el resumen del informe Horizon de 2019, publicado por el Instituto Nacional de Tecnologías Educativas y de Formación de Profesorado (INTEF), se presenta la formación del profesorado como uno de los retos más difíciles de abordar en el proceso de digitalización educativa y se reclama a las instituciones la creación de medidas de apoyo al profesorado sin limitaciones de ubicación o tiempo.

2 http://tcg.uis.unesco.org/survey-education-covid-school-closures/ 


\section{La CDD: definición y documentos de referencia}

Hace unos años, el desarrollo profesional docente pasaba por centrarse en los conocimientos sobre la materia objeto de impartición y en las teorías pedagógicas imperantes, así como en su habilidad para transferir esos conocimientos a la realidad del aula. En estos momentos, es evidente que para un óptimo desarrollo profesional docente es imprescindible sumar la competencia digital. En este sentido, el Consejo de Europa propone:

- Aprender de la crisis de la COVID-19, durante la cual la tecnología se está utilizando a una escala sin precedentes en educación y capacitación.

- Que los sistemas de educación y formación se adapten a la era digital.

Para ello, se han elaborado documentos con el objetivo de ayudar a los docentes a desarroIlar la CDD, como el Marco Común de Competencia Digital Docente (MCCDD), elaborado por el INTEF (2017). En este documento se concibe la CDD como el conjunto de conocimientos, habilidades y capacidades relacionadas con la tecnología en contextos educativos con el fin de alcanzar los objetivos propuestos en los procesos de enseñanza, aprendizaje y evaluación. La importancia del desarrollo de esta competencia digital entre el colectivo docente viene dada por la responsabilidad que este tiene en el desarrollo, a su vez, de dicha competencia entre los ciudadanos del futuro. Se entiende el MCCDD como un documento de referencia en constante actualización de las seis áreas que contempla en la formación del profesorado para que se pueda desarrollar, a su vez, la competencia digital del alumnado (véase figura 1).

Figura 1. Visión general del marco DigCompEdu

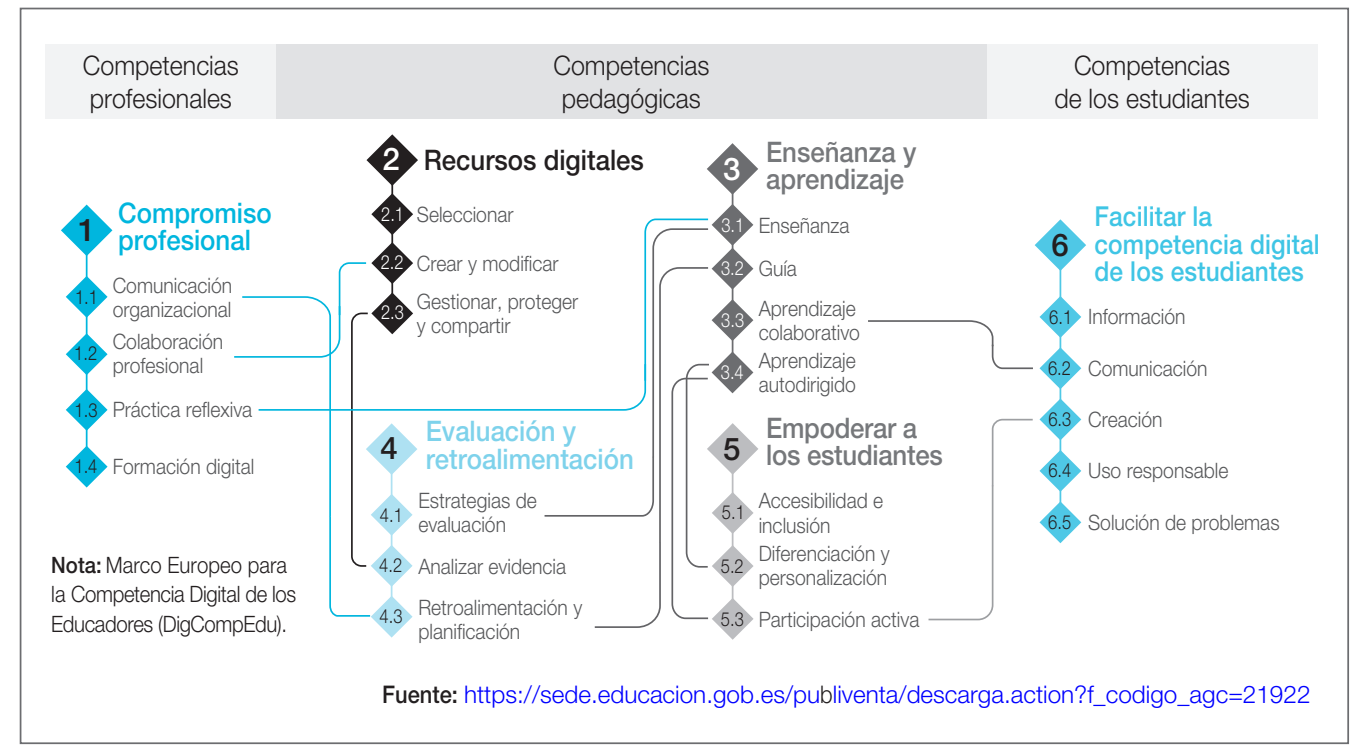


Se espera que este desarrollo competencial se aplique tanto en la formación inicial como en la formación continua del profesorado para, posteriormente, acreditar su nivel de competencia de acuerdo a los descriptores competenciales, divididos en seis niveles (véase figura 2).

Figura 2. Progresión de la competencia

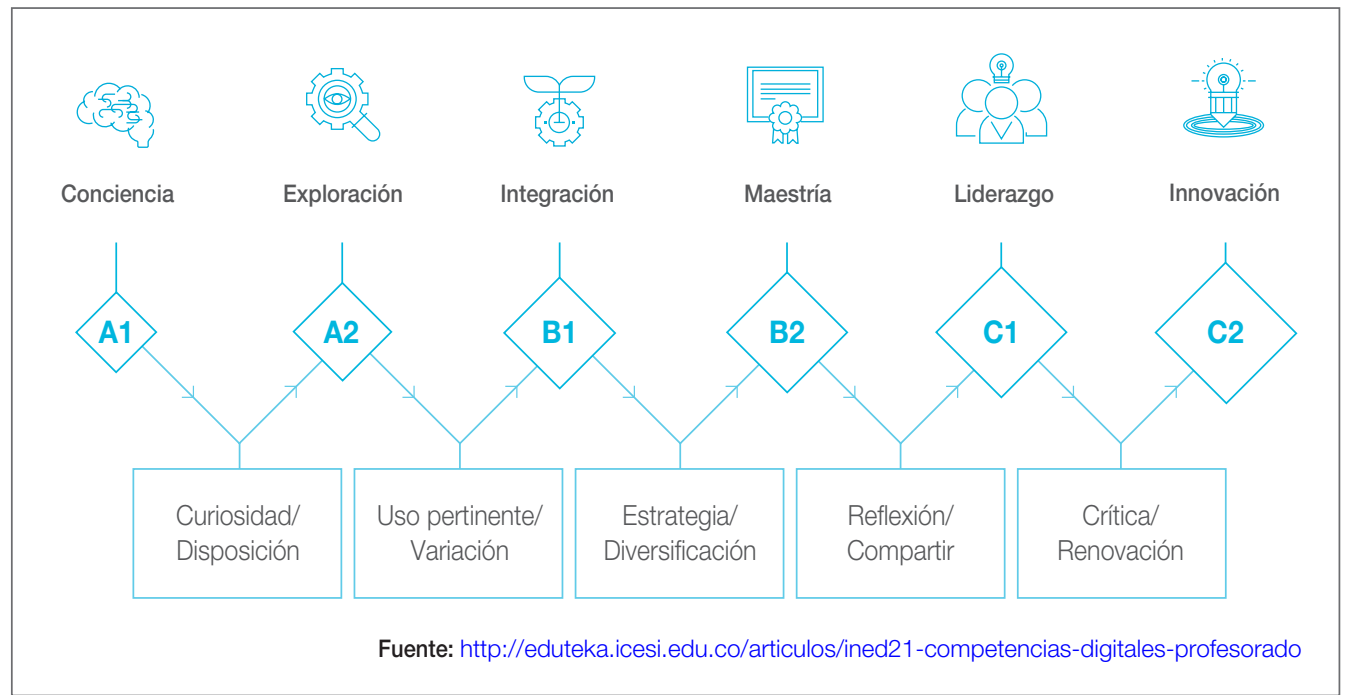

Los descriptores permiten al profesor hacerse una idea de cuáles son sus fortalezas en relación con la CDD y sus carencias para poder proponerse un plan de formación ajustado a sus necesidades digitales y a su contexto educativo.

\subsection{La autoevaluación de la CDD}

Otro de los proyectos de desarrollo de competencia digital que actualmente maneja Europa es el DigCompEdu ${ }^{3}$, elaborado por el Joint Research Centre (JRC) de la Comisión Europea, en el que se cuenta con antecedentes de varios proyectos europeos, tales como el DigEuLit: A European Framework for Digital Literacy (Martin y Grudzlecki, 2006) y el DigComp: A Framework for Developing and Understanding Digital Competence in Europe (Ala-Mutka, 2011; Ferrari, 2013). En él se propone un cuestionario, a modo de autoevaluación, para el desarrollo de la CDD con el objetivo de que el docente pueda averiguar en qué nivel se encuentra en relación con su CDD. Tras contestar los ítems que se presentan divididos en seis áreas,

3 https://ec.europa.eu/eusurvey/runner/DigCompEdu-H-ES?startQuiz=true\&surveylanguage=EN 
correspondientes a los niveles descritos, el sistema ofrece un informe del nivel, en relación con las diferentes competencias, y sugerencias para continuar desarrollando la competencia digital hasta alcanzar el grado máximo. Si bien es un cuestionario pensado para docentes universitarios, las preguntas y situaciones que se plantean son transferibles a otros contextos docentes.

\subsection{SELFIE: programa europeo para la competencia digital de la comunidad educativa}

En el contexto de la Comisión Europea se ha desarrollado una herramienta gratuita, SELFIE (reflexión personal sobre un aprendizaje efectivo mediante el fomento de la innovación a través de tecnologías educativas), con el fin de que los centros escolares integren las tecnologías digitales en sus procesos de enseñanza, aprendizaje y evaluación. SELFIE, a través de preguntas y afirmaciones breves para el alumnado, los docentes y el equipo directivo, permite obtener información objetiva y precisa sobre el estado de integración de la tecnología en los centros. A partir de los resultados recogidos, la plataforma elabora un informe sobre los puntos fuertes y débiles de los mismos con el fin de que puedan diseñar planes de formación y digitalización adecuados. Cualquier centro interesado en realizar una radiografía de su «digitalización» puede registrarse gratuitamente e iniciar así el proceso de autorreflexión. Una vez finalizadas las preguntas, el centro recibe un informe personalizado. Conviene asimismo destacar que todas las respuestas son anónimas y que los datos recopilados no se utilizarán con fines clasificatorios de los centros educativos participantes.

\subsection{El modelo TPACK como marco para el desarrollo de la competencia digital}

Con anterioridad al modelo presentado por el Consejo de Europa ya hubo modelos pedagógicos, a modo de marcos de referencia, para la mejora de la práctica docente. Así, Gudmundsdottir y Shulman propusieron en 1987 que la pedagogía debía relacionarse estrechamente con la materia objeto de impartición, a partir de su modelo PCK (pedagogical content knowledge). Koehler y Mishra (2009), siguiendo a Gudmundsdottir y Shulman, añadieron el ámbito tecnológico, desarrollando así el modelo TPACK (technological pedagogical content knowledge). Este modelo, a modo de marco conceptual y orientación al profesorado, persigue integrar las nuevas tecnologías en el aula (Vallejo, 2013), a saber:

- Enseñar con tecnología es un problema complejo.

- Los problemas complejos requieren de soluciones creativas. 
- Los profesores son diseñadores del paquete completo (PACKage), es decir, del modelo TPACK, que tiene en cuenta los tres elementos fundamentales: pedagogía, conocimiento sobre la materia y el uso de la tecnología.

Según estas premisas, los conocimientos pedagógicos, disciplinares y tecnológicos del docente se interrelacionan entre sí en el contexto del aula creando un modelo de diseño instruccional. El profesorado debe, pues, aprender a manejar sus conocimientos, habilidades y destrezas de manera que esta interacción suponga una mejora real de la calidad del

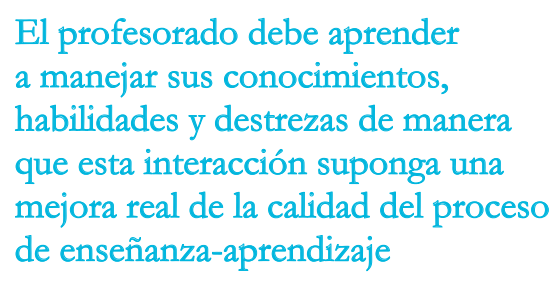

El profesorado debe aprender a manejar sus conocimientos, habilidades y destrezas de manera que esta interacción suponga una mejora real de la calidad del proceso de enseñanza-aprendizaje proceso de enseñanza-aprendizaje, integrando para ello los tres ámbitos descritos anteriormente. El modelo presenta siete áreas de conocimientos integrados a partir de los saberes pedagógicos, disciplinares y tecnológicos. Todos estos conocimientos deben ser interpretados de forma individual y en interacción, como se muestra en la figura 3:

- Conocimiento pedagógico (pedagogical knowledge [PK]). Conocimiento sobre pedagogía, didáctica y métodos de enseñanza que debe poseer todo docente.

- Conocimiento del contenido (content knowledge [CK]). Se trata del conocimiento que el profesor debe tener de la materia que ha de impartir.

- Conocimiento tecnológico (technological knowledge [TK]). Todo lo relacionado con el conocimiento y el uso de las nuevas tecnologías.

- Conocimiento pedagógico del contenido (pedagogical content knowledge [PCK]). Integra el conocimiento de la disciplina y cómo enseñarla, y afecta al conocimiento pedagógico y disciplinar.

- Conocimiento tecnológico del contenido (technological content knowledge [TCK]). Se refiere al conocimiento del docente en materia de tecnologías y cuáles son las más adecuadas para enseñar su materia.

- Conocimiento tecnológico-pedagógico (technological pedagogical knowledge [TPK]). Se trata del conjunto de saberes relacionados con el uso de las nuevas tecnologías en educación.

- Conocimiento tecnológico-pedagógico del contenido (TPACK). Alude a la integración de todos los componentes anteriores en su conjunto y supone integrar el conocimiento del docente sobre la materia, la metodología más adecuada, con la tecnología más apropiada para mejorar la enseñanza de un contenido concreto. 
Figura 3. Modelo TPACK

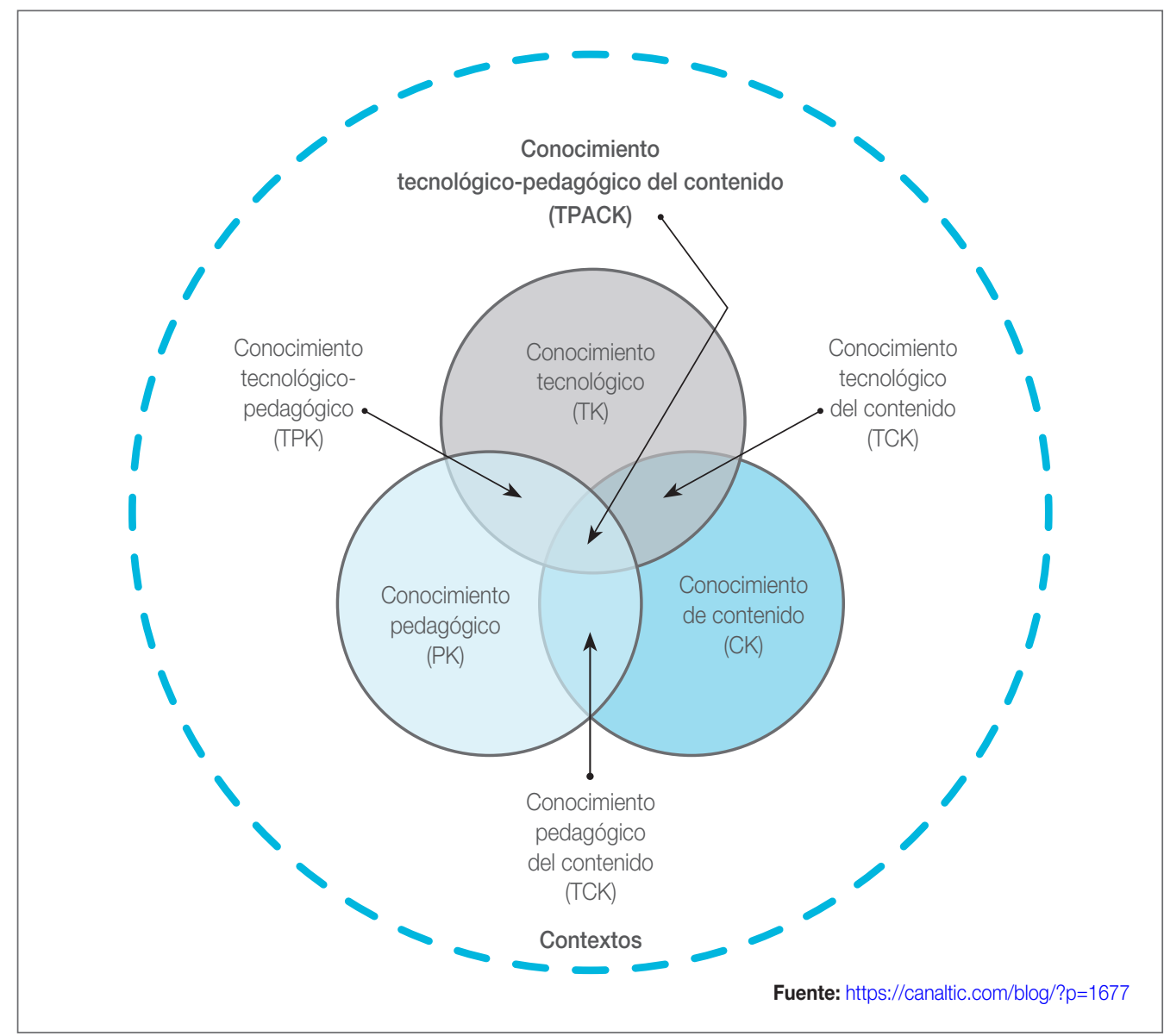

A fin de implementar correctamente este modelo, el equipo de trabajo formado por Harris y Hofer desarrolló una estrategia para aplicar el modelo TPACK, basada en un proceso de toma de decisiones de cinco estadios, tal como resume Vallejo (2013):

- Seleccionar los objetivos de aprendizaje de la materia concreta que se va a impartir.

- Determinar cómo van a ser las experiencias de aprendizaje.

- Seleccionar y secuenciar las actividades.

- Seleccionar las estrategias para la aplicación de la evaluación formativa y sumativa.

- Seleccionar tecnologías adecuadas para el desarrollo de las actividades propuestas. 
En el apartado 5.3.6 del presente artículo se sugiere una plantilla a modo de guía que sirva a los docentes en la planificación de sus sesiones conforme al modelo TPACK.

\section{La observación como herramienta para el desarrollo de la CDD}

Como ya se ha señalado en apartados anteriores, el desarrollo de la CDD pasa por saber en qué punto se encuentra el centro (cuestionario SELFIE) y el docente en relación con dicha competencia (cuestionario DigCompEdu), por ello, además de estos cuestionarios, es necesario contar con herramientas que nos aporten más datos sobre la realidad docente. Asimismo, se recomienda aprovechar el nuevo escenario de docencia online, derivado de la COVID-19, que permite a los profesores grabar sus clases y observar una realidad que les pa-

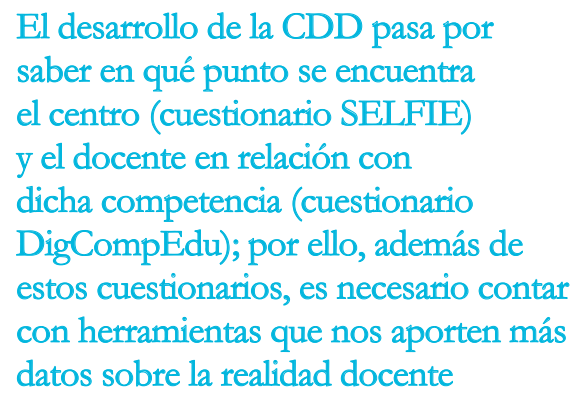

El desarrollo de la CDD pasa por saber en qué punto se encuentra el centro (cuestionario SELFIE) y el docente en relación con dicha competencia (cuestionario DigCompEdu); por ello, además de estos cuestionarios, es necesario contar con herramientas que nos aporten más datos sobre la realidad docente recía vedada. Esta posibilidad técnica de la grabación de clases implementadas en las nuevas plataformas, como Zoom, Meet, Blackboard Collaborate o bbbserver, entre otras, puede contribuir de manera eficaz al desarrollo de la CDD a partir de la observación de sus prácticas como una forma de recoger información sobre la enseñanza (Richards y Lockhart, 1998). Estas grabaciones pueden ser analizadas a través de la autoobservación y de la observación entre iguales, en el caso de la formación continua, o de la observación por parte de un profesorado experimentado o mentor, en el caso de la formación inicial.

Los centros decididos a diseñar un plan de observación en línea entre iguales que fomente el análisis y la evaluación pueden considerar los siguientes elementos observables en sus clases (Tobin et al., 2015). La lista que estos autores proponen es susceptible de ser modificada por cada centro para adaptar el plan a sus necesidades y objetivos:

- Estructura y manejo del espacio virtual de aprendizaje (EVA).

- Interacción docente-discentes.

- Maestría del área de conocimiento.

- Instrucción.

- Personalización del aprendizaje.

- Evaluación.

- Inclusión. 


\section{Propuesta de diseño de un plan de información a través de un CDCO}

Uno de los errores que se ha producido en el contexto actual ha sido replicar en el entorno online las mismas técnicas de gestión de aula y el mismo contenido de la clase presencial, sin considerar cuáles eran la metodología y el enfoque más adecuados. En el caso de la enseñanza en línea resulta imprescindible reflexionar sobre aspectos como la tipología y el tiempo de actividades, el potencial de la plataforma seleccionada, los instrumentos de evaluación, la presentación de los contenidos, la
Uno de los errores que se ha producido en el contexto actual ha sido replicar en el entorno online las mismas técnicas de gestión de aula y el mismo contenido de la clase presencial, sin considerar cuáles eran la metodología y el enfoque más adecuados para el nuevo contexto necesidad de incluir recreos mentales o la disponibilidad de dispositivos electrónicos y de conexión en los hogares y en los centros educativos. Esta nueva realidad ha evidenciado que la enseñanza online supone mucho más que emplear la tecnología con acceso a internet. A fin de que los docentes tengan éxito en este modelo de aula, necesitan saber cómo crear y presentar contenido motivador y asegurarse de que desarrollan en sus estudiantes la competencia digital necesaria. Aun teniendo en cuenta que ya existen instrumentos de evaluación para el modelo de observación en el contexto de aula tradicional, como fichas de observación, notas de campo, portafolios, diarios de clase, etc. (Montmany y González, 2019), cuando se trata del aula en línea, muchas instituciones han expuesto sus dudas sobre cómo deben diseñarse e implementarse los programas y métodos de evaluación. A continuación, compartimos algunas de las dudas más frecuentes en este sentido:

- ¿Cuál es el papel del observador? ¿Qué perfil de observador está más cualificado para observar una sesión en línea?

- ¿Se deben incluir elementos observables relacionados con la competencia digital y el manejo de los EVA en los cuestionarios de observación?

- ¿Cómo afectarán los problemas técnicos a la evaluación y al feedback del observado?

La propuesta que aquí se detalla intenta dar respuesta a estas y otras cuestiones, al tiempo que ofrece un modelo de plan de formación estándar, accesible y flexible, a modo de guía, y fácil de implementar por cualquier equipo docente interesado en crear una cultura de observación en su centro para el desarrollo de la CDD.

Junto con los elementos observables en relación con la práctica docente, el plan también cuenta con unos objetivos específicos encaminados a desarrollar la CDD, así como la del alumnado y demás agentes de la comunidad educativa. En este sentido, las áreas de 
desarrollo recogidas en el documento oficial del MCCDD de los Educadores (DigCompEdu) son las que han sido empleadas como referencia a la hora de trazar los objetivos de nuestra propuesta (véase apartado «La CDD: definición y documentos de referencia»).

\subsection{Destinatarios}

El plan de observación propuesto pretende ser flexible y fácilmente adaptable a cualquier contexto educativo, desde equipos reducidos de docentes de centros de enseñanza no reglada, hasta equipos multidisciplinares de centros educativos de carácter público y privado cuyo objetivo sea implantar un plan de observación de centro para la mejora de la CDD, crear una cultura de evaluación y proporcionar un entorno dinámico de formación continua. Para su diseño se necesita que el centro y el equipo implicado se familiaricen con la propuesta y establezcan unos criterios y unos elementos de observación basados en sus necesidades específicas y en los recursos digitales a su disposición.

\subsection{Metodología}

La investigación-acción (action-research) se trata de un enfoque cuyo principal objetivo es entender la naturaleza de problemas complejos, sociales u organizacionales a través de la reflexión y la implementación de acciones tomadas en función de los resultados observados. Este método ha sido ampliamente aceptado y utilizado exitosamente en campos como la educación (Carr y Kemmis, 1986), entre otros.

Este enfoque se compone de cuatro fases principales:

- Planificación de la acción.

- Acción.

- Observación.

- Reflexión.

Cada ciclo aporta nuevos conocimientos y aprendizaje sobre la organización, sus problemas y posibles soluciones, así como sobre los diferentes campos de conocimiento involucrados. La repetición del proceso cíclico aporta información desde distintas perspectivas en relación con un aspecto específico para la comprensión más completa de un problema general y sus posibles soluciones. En la figura 4 se observan las relaciones que es posible establecer entre los momentos principales del proceso, los cuales pretenden reducir la distancia entre el conocimiento y la acción, así como dotar al profesorado de recursos para mejorar su práctica docente. 
Figura 4. Modelo de creación de ciclos de planificación, acción, observación y reflexión

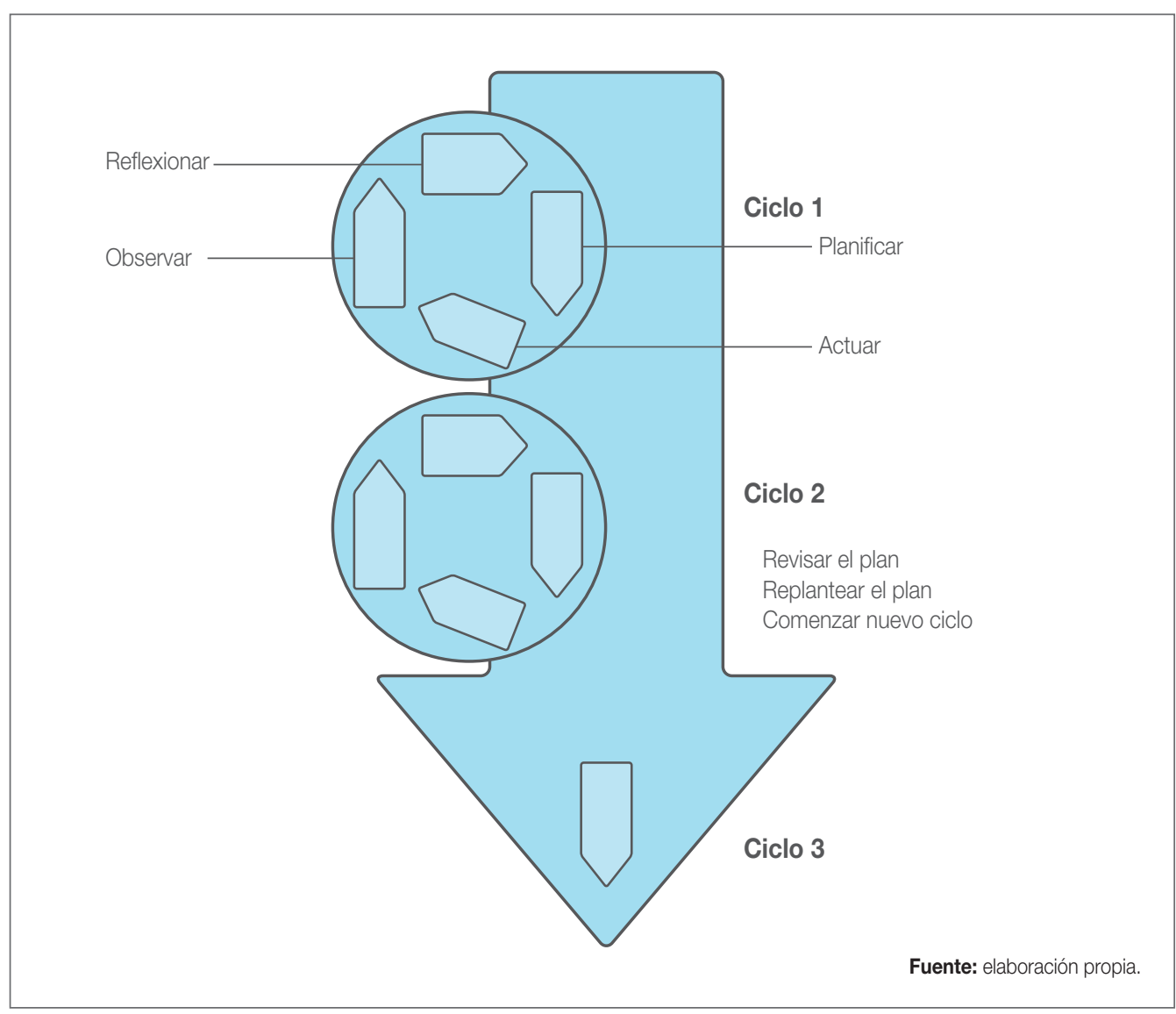

En este modelo se plantea que el profesor, para realizarse y emanciparse en su ejercicio profesional, debe generar sistemáticamente ciclos de:

- Planificación. Oganización de la acción docente partiendo de las seguridades e inquietudes personales y profesionales.

- Acción. Puesta en práctica de las ideas que ha planificado previamente en la enseñanza con su(s) grupo(s) de estudiantes.

- Observación. Grabación, diario, etc., de la acción, además de la reflexión que se produce durante el proceso de acción.

- Reflexión. Análisis posterior a la observación realizada, «reflexión sobre la acción», con el objeto de iniciar una nueva planificación y un nuevo ciclo de investigaciónacción. 
Este enfoque otorga un gran protagonismo al docente y considera que será su autonomía investigadora la que le permita desarrollarse profesionalmente. Asimismo, este enfoque fomenta la relación y las interacciones entre el profesorado, además de favorecer el intercambio de ideas y reflexiones a través del trabajo con herramientas colaborativas.

\subsection{Perfil del profesorado observador}

El observador ha de ser un profesor formado y con experiencia en el tipo de clases que vaya a observar, en el enfoque adoptado, en el nivel y en el contexto de enseñanza, con el fin de ofrecer feedback formativo. Ha de tener capacidad de captar todas las acciones que se desarrollan de forma simultánea en el aula y a la vez poder aislarlas en función de los objetivos marcados. Además, observar clases online implica poseer una óptima CDD con el fin de conocer el potencial de las diferentes plataformas y aplicaciones digitales para la enseñanza y así poder responder a las dudas e inquietudes de los observados.

El papel del observador consiste, en una primera fase, en ayudar al docente a organizar la observación, acotando los objetivos de la misma y recogiendo información sobre el grupo, el programa, el enfoque y el entorno digital de trabajo. En la fase propiamente de observación su rol es el de anotar la información en las fichas propuestas. La última fase consiste en el encuentro con el observado. Para ello ha de preparar sus fichas de observación, sus notas y comentarios, buscar los aspectos positivos de lo que ha observado, los aspectos mejorables y las alternativas para estos, y así poder ofrecer retroalimentación de calidad.

\section{Cuaderno Digital Colaborativo de Observación (CDCO)}

En este apartado presentamos el CDCO creado con OneNote, sus objetivos y las diferentes partes en que se ha estructurado («Manual de usuario», «Diagnóstico de centro», «Diagnóstico CDD», «Acción», «Observación», «Planificación», «Reflexión», «Caja de herramientas» y «Ejemplo de caso») para dar cabida al plan de observación completo con el que desarrollar la CDD (véase figura 5). 
Figura 5. Presentación del CDCO

CDCO Manual de usuario Diagnóstico del centro Diagnóstico CDD Planificación Acción Observación Reflexión Mis herramientas Ejemplo de caso ++

\section{Mi cuaderno digital colaborativo de observación}

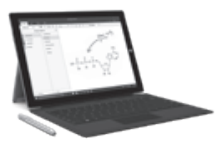

El CDCO pretende ser una guía flexible y adaptable para todos aquellos docentes que deseen implementar una cultura de observación

en sus centros con el objetivo de mejorar su actividad docente y desarrollar la competencia digital en contexto a través de la observación en línea y el análisis reflexivo consciente de su EVA.

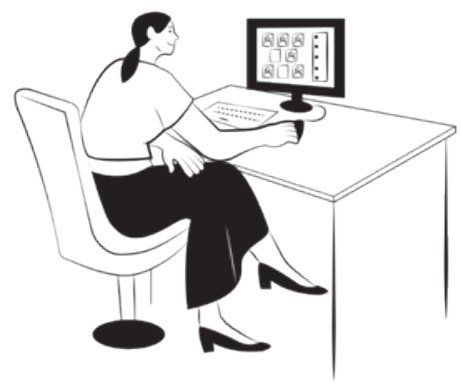

Fuente: https://rb.gy/gkrbnw

Este cuaderno se ha diseñado a partir de los conceptos y documentos de referencia presentados en apartados anteriores, siendo coherente con los consejos que estos ofrecen para el óptimo desarrollo de la CDD.

Aunque a continuación se realiza un resumen detallado del contenido del cuaderno, también puede accederse a la versión completa en línea (véase nota a pie de página) ${ }^{4}$.

\subsection{Justificación de la selección de la herramienta OneNote}

Con el objetivo de crear un plan de observación comprensivo que facilite la formación y el desarrollo en contexto de la competencia digital de los diferentes agentes de la comunidad educativa, tal como se ha venido defendiendo en apartados anteriores, se propone el diseño y la implementación de un CDCO fácilmente editable, flexible y versátil con OneNote, una herramienta de acceso gratuito desarrollada por Microsoft para su paquete Office.

4 https://1drv.ms/u/s!AmJ5055N5CY5jioB-agxbsv_L3xu?e=qwNcrx 
OneNote permite la toma de notas, la recopilación de información y la colaboración multiusuario en un único espacio, lo cual supone un excelente marco para la interacción de los diferentes agentes de la comunidad educativa en un contexto seguro y privado.

Esta herramienta es fácil de usar al permitir organizar el contenido en una aplicación que lo sincroniza con la nube automáticamente. Además, estos datos pueden verse en tiempo real desde la propia aplicación web de OneNote o, si se prefiere, también se pueden sincronizar en el ordenador, tanto de Windows como de Mac, instalando la aplicación de escritorio. También es posible descargar la aplicación para smartphone y tableta.

Las razones fundamentales para elegir OneNote frente a otras herramientas de observación más consolidadas como VEO 5 son, principalmente, que se trata de un recurso que va más allá de la mera recogida de datos y de la grabación de la sesión durante el proceso de observación. Gracias a las prestaciones que ofrece OneNote se ha podido diseñar un cuaderno digital con pautas adaptadas, cuestionarios para la autoevaluación, fichas, infografías, protocolos de observación y herramientas para evaluar y desarrollar la competencia digital, promoviendo así la colaboración en remoto, pues el desarrollo de la CDD debe trascender más allá de la formación individual del profesorado en materia de tecnologías de la información y la comunicación (TIC), siendo necesario el desarrollo de prácticas docentes generadoras, integradoras y colaborativas que tengan en cuenta a todos los miembros que forman parte de la comunidad educativa (Castañeda et al., 2018).

\subsection{Objetivos}

A continuación, se detallan los principales objetivos de formación en relación con el desarrollo de la CDD que es posible alcanzar en el contexto de uso del CDCO (véase figura 6).

5 https://veo.co.uk/
OneNote permite la toma de notas, la recopilación de información y la colaboración multiusuario en un único espacio, lo cual supone un excelente marco para la interacción de los diferentes agentes de la comunidad educativa en un contexto seguro y privado. Es una herramienta fácil de usar

Gracias a las prestaciones que ofrece OneNote se ha podido diseñar un cuaderno digital con pautas adaptadas, cuestionarios para la autoevaluación, fichas, infografías, protocolos de observación y herramientas para evaluar y desarrollar la competencia digital, promoviendo así la colaboración en remoto, pues el desarrollo de la CDD debe trascender más allá de la formación individual del profesorado en materia de TIC 
Figura 6. Objetivos del CDCO

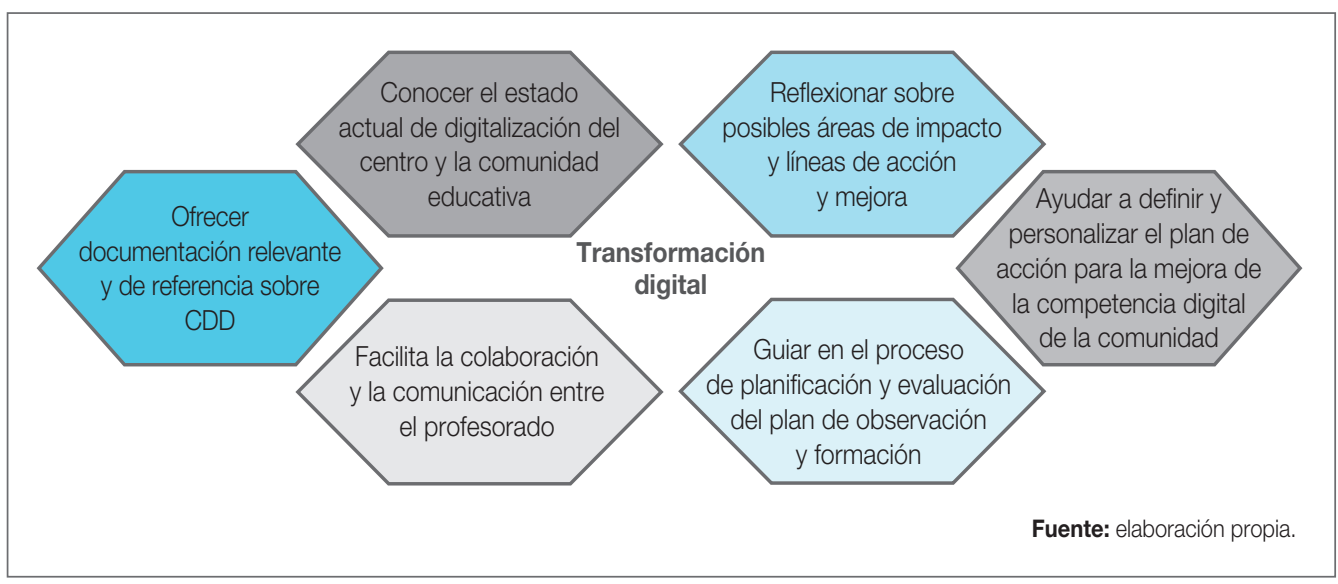

Los objetivos "Conocer el estado actual de digitalización del centro y de la comunidad educativa», "Reflexionar sobre posibles áreas de impacto y líneas de acción y mejora» y «Ayudar a definir y personalizar el plan de acción para la mejora de la competencia digital de la comunidad» se cubren de forma específica con los apartados «Diagnóstico de centro» $y$ «Diagnóstico CDD» del CDCO a partir de los cuestionarios SELFIE y del cuestionario del docente facilitado por DigCompEdu. El objetivo «Guiar en el proceso de planificación y evaluación del plan de observación y formación» queda cubierto con los apartados centrales del CDCO («Planificación», «Acción», «Observación», «Reflexión»y «Ejemplo de caso»).

En cuanto al objetivo «Facilitar la colaboración y comunicación entre el profesorado», este quedaría justificado con la herramienta seleccionada: OneNote. Esta herramienta de Microsoft permite trabajar de modo colaborativo entre los usuarios a los que se les haya dado acceso, facilitando de ese modo la comunicación y el seguimiento del proceso de formación.

El objetivo «Ofrecer documentación relevante y de referencia sobre la CDD» se garantiza con la información ofrecida en el apartado «Mis herramientas» del CDCO. Este apartado, además de alojar los documentos de referencia de la Comisión Europea, incorpora cápsulas formativas y apps útiles para el desarrollo de la competencia digital de los docentes y del alumnado.

\subsection{Contenido}

EI CDCO estructura su contenido a partir de varias pestañas según el estado actual de la CDD que se ha presentado hasta ahora, yendo desde el diagnóstico de la competencia digital del centro hasta un plan de observación individual con sus fases de desarrollo y su evaluación. El usuario solo tendrá que hacer un duplicado o copia en su dispositivo para irlo desarrollando o compartir el mismo cuaderno con otros usuarios. 


\subsubsection{Manual de usuario}

Este primer apartado sirve de ayuda al docente que se inicia en procesos de observación para su propia formación y facilita la navegación por el CDCO. En él, se presentan las diferentes páginas y subpáginas que lo componen, las tareas que se espera que el docente realice en cada una de ellas y los objetivos específicos que persiguen las tareas propuestas (véase figura 7).

Figura 7. «Manual de usuario» del CDCO

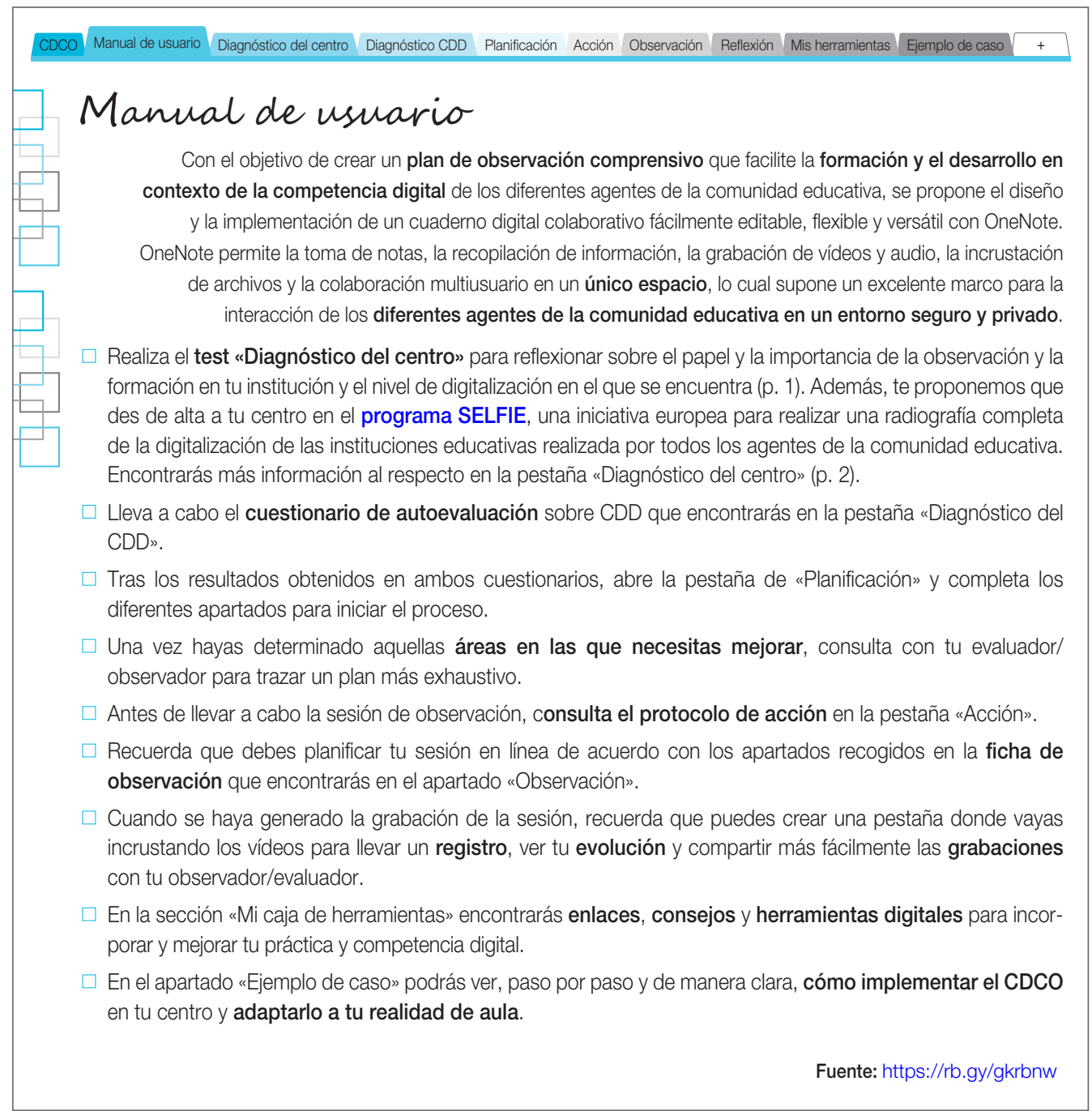




\subsubsection{Diagnóstico del centro}

Iniciar un plan de desarrollo de la CDD ha de partir de un análisis de necesidades del centro para calibrar de forma realista la magnitud del plan al que el docente ha de enfrentarse. Recordemos que para recoger información de los diferentes agentes implicados en la docencia y en la gestión del centro (alumnado, docentes y personal directivo) disponemos de herramientas como SELFIE (véase apartado 2.2.) que pueden facilitarnos la labor, al guardar nuestro registro para que podamos acceder a él tantas veces como sea necesario. Mostramos la página del CDCO destinada a ese objetivo (véase figura 8).

Figura 8. "Diagnóstico del centro» del CDCO (apartado "SELFIE»)

CDCO Manual de usuario Diagnóstico del centro Diagnóstico CDD Planificación Acción Observación Reflexión Mis herramientas Ejemplo de caso +

SELFIE - Descubre el potencial digital de tu centro

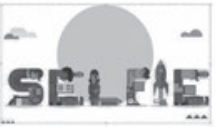

SELFIE recoge, de forma anónima, las opiniones del alumnado, del profesorado y del personal directivo de los centros educativos sobre el modo en que se utiliza la tecnología en el centro. Para ello se utilizan preguntas y afirmaciones breves y una escala simple de valoración del 1 al 5. Sobre la base de esta información, la herramienta genera un informe, una instantánea (un selfie) de los puntos fuertes y débiles del uso de la tecnología que hace el centro educativo. SELFIE está disponible para cualquier centro de enseñanza primaria, secundaria y profesional en Europa y fuera de ella, y en más de 30 lenguas. Puede ser utilizado por cualquier centro, y no únicamente por los centros con un nivel avanzado de infraestructuras, equipamiento y uso de tecnologías.

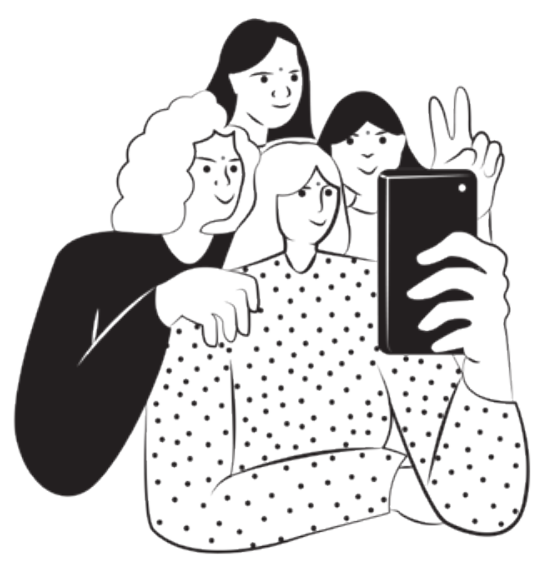


Enfrentarse a este tipo de cuestionarios implica iniciar procesos de reflexión que ayuden a tomar conciencia de la necesidad de llevar a cabo el plan de desarrollo propuesto. En este apartado también se propone el test «Diagnóstico del centro» a fin de invitar a los diferentes miembros de la comunidad a reflexionar sobre el papel y la importancia de la observación y la formación en la institución a la que pertenecen y al nivel de digitalización en el que se encontraría (véase figura 9).

Figura 9. «Diagnóstico del centro» del CDCO (apartado «Cuestionario»)

\begin{tabular}{|c|c|c|c|c|}
\hline CDCO Manual de usuario Diagnóstico del centro Diagnóstico CDD Planificacic & n Acción 0 & iervación Reflexión & Mis herramientas $\mathrm{Ej}_{\mathrm{f}}$ & nplo de caso \\
\hline \multicolumn{5}{|c|}{ Cuestionario para el diagnóstico del centro } \\
\hline \multicolumn{5}{|c|}{ Indica en qué medida estás de acuerdo con las siguientes afirmaciones: } \\
\hline A día de hoy, mi centro cuenta con... & $\begin{array}{l}\text { Muy de } \\
\text { acuerdo }\end{array}$ & De acuerdo & En desacuerdo & $\begin{array}{c}\text { En total } \\
\text { desacuerdo }\end{array}$ \\
\hline \multicolumn{5}{|l|}{$\begin{array}{l}\text { Un plan para la observación en el contexto de aula tra- } \\
\text { dicional. }\end{array}$} \\
\hline \multicolumn{5}{|l|}{$\begin{array}{l}\text { Un método para la comunicación de los resultados de } \\
\text { evaluación de la observación a los docentes. }\end{array}$} \\
\hline \multicolumn{5}{|l|}{$\begin{array}{l}\text { Un método para la comunicación de los resultados de } \\
\text { las encuestas de satisfacción de los estudiantes. }\end{array}$} \\
\hline \multicolumn{5}{|l|}{$\begin{array}{l}\text { Herramientas específicas para recoger información so- } \\
\text { bre el proceso de enseñanza. }\end{array}$} \\
\hline \multicolumn{5}{|l|}{ Una cultura de formación docente. } \\
\hline \multicolumn{5}{|l|}{$\begin{array}{l}\text { Un sistema para la autoevaluación de las prácticas do- } \\
\text { centes. }\end{array}$} \\
\hline \multicolumn{5}{|l|}{ Un sistema para la evaluación informal entre iguales. } \\
\hline \multicolumn{5}{|l|}{$\begin{array}{l}\text { Una cultura en la que el equipo docente realiza cambios } \\
\text { a la actividad docente basándose en los resultados de } \\
\text { las encuestas de los estudiantes y en la autoevaluación. }\end{array}$} \\
\hline \multicolumn{5}{|l|}{ Un plan para la observación en línea. } \\
\hline \multicolumn{5}{|l|}{ Profesorado con experiencia docente en línea. } \\
\hline \multicolumn{5}{|l|}{$\begin{array}{l}\text { Información detallada sobre el acceso a dispositivos elec- } \\
\text { trónicos y conexión a internet por parte del alumnado. }\end{array}$} \\
\hline \multicolumn{5}{|l|}{$\begin{array}{l}\text { Información detallada sobre el nivel real de competencia } \\
\text { digital de los docentes. }\end{array}$} \\
\hline Acceso a un EVA propio. & & & & \\
\hline
\end{tabular}

Figura I: Checklist traducida y adaptada de Tobin et al. (2015). Evaluating Online Teaching: Implementing Best Practices.

Fuente: https://rb.gy/gkrbnw 


\subsubsection{Diagnóstico CDD}

Una vez elaborado el diagnóstico del centro, ya podemos centrarnos en nuestras necesidades específicas como docentes. Al contestar el cuestionario específico para la autoevaluación de la CDD (véase apartado 2.1), obtendremos un informe detallado que nos informará de nuestras fortalezas y puntos débiles, a la vez que nos ofrecerá sugerencias para continuar nuestro desarrollo (véase figura 10).

Figura 10. «Diagnóstico CDD» del CDCO

CDCO Manual de usuario Diagnóstico del centro Diagnóstico CDD Planificación Acción Observación Reflexión Mis herramientas Ejemplo de caso +

\section{Competencias digitales}

\section{Test de competencias digitales}

Esta herramienta de autoevaluación se basa en el Marco Europeo de Competencia Digital para Educadores (DigCompEdu). DigCompEdu establece 22 competencias organizadas en seis áreas. Las competencias se explican en seis niveles diferentes de habilidad (A1, A2, B1, B2, C1 y C2). DigCompEdu se dirige a educadores en todos los niveles de educación, desde preescolar hasta educación primaria, secundaria y superior. El marco está enfocado en apoyar y motivar a los profesores en el uso de herramientas digitales para mejorar e innovar en la educación.

Esta herramienta tiene como objetivo permitirle reflexionar sobre sus fortalezas y debilidades en el uso de las tecnologías digitales en la educación. Le invitamos a autoevaluarse con 22 elementos que son representativos de las 22 competencias del marco DigCompEdu. Para cada uno de estos elementos, elija una de las cinco opciones de respuesta.

\section{Accede al test aquí}

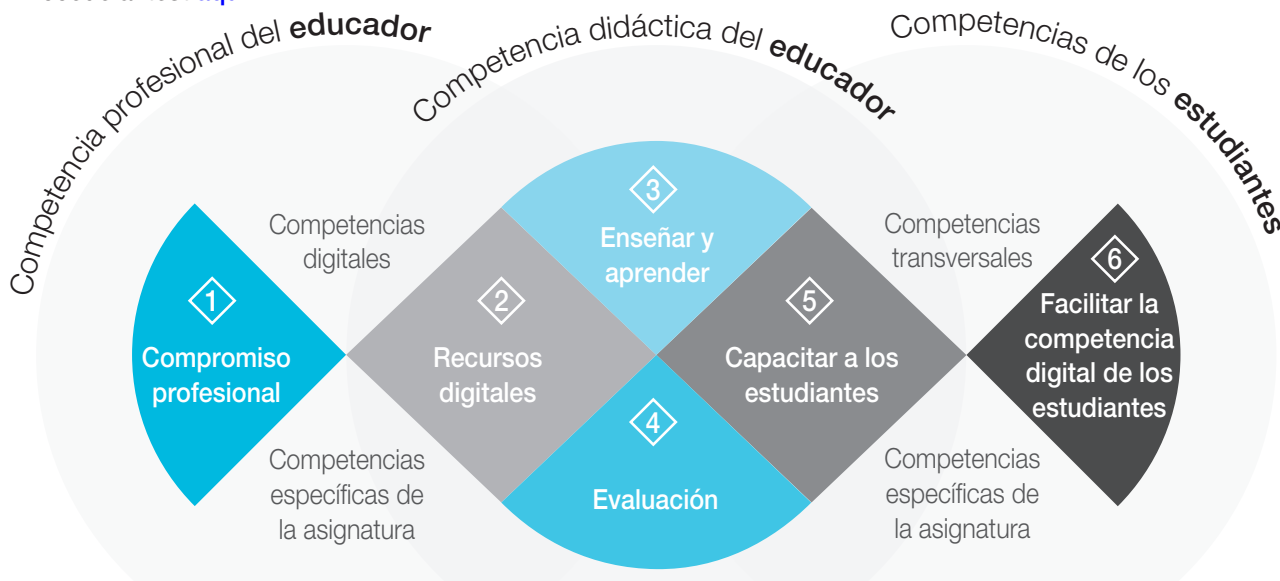

Fuente: https://ec.europa.eu/eusurvey/runner/DigCompEdu-H-ES?startQuiz=true\&surveylanguage=EN

Fuente: https://rb.gy/gkrbnw 
Poder acceder a la tecnología y tener competencia para usarla en el ámbito privado no garantiza saber transferir esa competencia al ámbito profesional, por ello la realización de este tipo de cuestionarios nos ayudará a tomar conciencia de las posibilidades que existen.

\subsubsection{Planificación}

Llevar a cabo un plan de desarrollo profesional no es una tarea simple, es por ello por lo que el docente ha de organizarse partiendo de las seguridades e inquietudes personales y profesionales en relación con su competencia digital. Para poder cubrir esa tarea, el CDCO ofrece al docente una serie de pautas que le pueden ayudar a organizar tanto los objetivos que se plantea alcanzar como el cronograma para llevarlo a cabo. Las pautas reflejadas pueden dar pistas al profesor sobre las herramientas de trabajo que puede utilizar y cómo aplicarlas (véase figura 11).

Figura 11. «Planificación» del CDCO

CDCO Manual de usuario Diagnóstico del centro Diagnóstico CDD Planificación Acción Observación Reflexión Mis herramientas Ejemplo de caso +

\section{Planificación}

Organización de la acción docente partiendo de las seguridades e inseguridades personales y profesionales en relación con la competencia digital.

Conocida la realidad y habiendo delimitado el problema, se debe establecer el plan de acción que se va a llevar a cabo. Es importante recordar que la competencia digital, como cualquier otra competencia, se ha de ir desarrollando de forma progresiva, por lo que el ámbito de actuación en torno al que gire el problema delimitado no ha de ser muy ambicioso para asegurarnos el éxito. No se debe olvidar que dicho plan no se entiende como algo totalmente cerrado y delimitado. Si algo caracteriza a la I-A es una estructura abierta y flexible. El plan general que se elabore debe ser lo suficientemente flexible como para que pueda incorporar aspectos no previstos en el transcurso de la investigación que podrán ser integrados en las acciones ya establecidas:

\section{Plantear problemática}

- En relación con mi competencia digital me preocupa...

- Siento que necesito mejorar...

Delimitar objetivos y organizar la secuencia de actuación

- Voy a concentrarme en los siguientes objetivos...

- Voy a seguir los siguientes pasos...

Describir cómo se van a controlar las

mejoras generadas por la investigación

- Durante el proceso llevaré un diario donde anotaré..

- Me serviré del dispositivo móvil para...
Establecer un cronograma realista de las acciones que se van a llevar a cabo

- Utilizaré Google Calendar para establecer fechas clave con mi supervisor.

- ¿Tutorías semanales?

Establecer cómo se van a recoger los datos

- ¿Grabación de las sesiones en vídeo?

- ¿Registro en vídeos/audios de las notas tomadas?

- ¿E-porfolio?

- ¿Notas en papel?

Fuente: https://rb.gy/gkrbnw 
Es importante dedicarle tiempo a esta fase de planificación, ser consciente de los recursos $y$, sobre todo, ser realistas. En este sentido, se han de proponer metas viables y realizables a corto plazo si queremos asegurar el éxito en nuestro plan de desarrollo profesional.

\subsubsection{Acción}

Este espacio tiene el objetivo de ayudar al docente a realizar una revisión de todo lo que necesitará para la puesta en práctica de las ideas planificadas previamente para la sesión de clase en la que se llevará a cabo la observación de la práctica docente y el dominio de la competencia digital: la revisión del material, la plataforma, la conexión, facilitarle acceso al observador, etc. También es el momento de tomar conciencia de la importancia de quién va a observar. En este sentido, se pueden distinguir diferentes tipos de observación en función de quién es la persona a la que se observa: el docente observándose a sí mismo (autoobservación) o un tutor o mentor con el fin de ayudar al observado a establecer relaciones entre los aspectos teóricos relacionados con su formación y las cuestiones prácticas y técnicas específicas de la actividad docente. No siempre resulta tarea fácil encontrar momentos en que el observador esté disponible para observar nuestras sesiones, por lo que una buena previsión nos ayudará a optimizar el tiempo de ambos (véase figura 12).

Figura 12. «Acción» del CDCO

CDCO Manual de usuario Diagnóstico del centro Diagnóstico CDD Planificación Acción Observación Reflexión Mis herramientas Ejemplo de caso +

\section{Acción}

Puesta en práctica de las ideas planificadas previamente para la enseñanza con los grupos de estudiantes

El proceso de investigación-acción se planifica con la finalidad de intervenir y poner en marcha cambios que modifiquen la realidad estudiada, en nuestro caso el nivel de competencia digital. Las acciones que vamos a llevar al aula son las que se corresponden con nuestros objetivos de desarrollo profesional, las que vamos a observar y, posteriormente, a analizar. Es importante que todo quede recogido en la planificación de nuestra sesión de clase y en la ficha de observación que elaboremos. Partiremos de la premisa de que los datos recogidos con los diversos instrumentos, por sí mismos, no son suficientes para establecer relaciones, analizar y extraer significados relevantes de cara al problema abordado. Se necesita contextualizar su análisis con un sentido secuencial, ya que, junto a la descripción de situaciones educativas (en las observaciones, entrevistas y diarios), están los juicios, las opiniones, las dudas, las reflexiones y las interpretaciones del investigador, haciendo necesario que el análisis y la elaboración de los datos se alternen.

Mi protocolo de acción

Tengo preparada y lista la herramienta o plataforma con la que grabaré la sesión.

(9) $\square$ Soy consciente de las áreas en las que seré evaluado por mi observador.

1 Me he asegurado de que la plataforma de videoconferencia y demás apps que utilizaré durante la sesión funcionan correctamente.

(I) $\square$ He repasado mi plan de clase y comprobado que se corresponde con los apartados de la ficha de observación.

(a) He facilitado el acceso al EVA y a la sesión en línea a mi evaluador/observador.

こ $\square$ Me he asegurado de que todos los estudiantes tienen buena conexión y el enlace de acceso a la herramienta de videoconferencia. 


\subsubsection{Observación}

Esta página dispone de un espacio en el que se le ofrecen ideas al docente para facilitar la observación, tales como elaborar una planificación de la sesión de clase basada en el modelo TPACK, descrito en el apartado 2.3 del presente artículo, y la cual permite recoger, de forma clara y sencilla, toda la información sobre los objetivos propuestos, las características del grupo, los agrupamientos, las actividades que se van a realizar, etc. Además, se incorporan cuatro pestañas con los siguientes apartados: «Para facilitar la observación», «Protocolo de observación», «Mi rúbrica de observación y evaluación» (tiene el objetivo de evaluar criterios de observación y evaluación del proceso a fin de orientar al profesorado en cuanto a la selección y utilización de las tecnologías en los proyectos docentes) y «Propuesta de fichas para la observación», las cuales pueden servir a modo de ejemplo para que el profesor y el observador diseñen sus propias fichas según los objetivos trazados (véanse figuras 13 a 16).

Figura 13. «Observación» del CDCO (apartado «Para facilitar la observación»)

\begin{tabular}{|c|c|c|c|}
\hline $\begin{array}{c}\text { Grupo } \\
\text { (Nivel) (Fecha) }\end{array}$ & $\begin{array}{l}\text { Define el grupo para el que se } \\
\text { va a planificar la sesión. } \\
\text { Anticipa posibles problemas } \\
\text { que puedan surgir con el grupo } \\
\text { meta: técnicos, de gestión de } \\
\text { aula, de disciplina... }\end{array}$ & Procedimientos & $\begin{array}{l}\text { Dinámicas, secuenciación, temporalización } \\
\text { y agrupación: } \\
\text { - Especifica cuáles van a ser las principales } \\
\text { dinámicas que van a componer la sesión } \\
\text { y su finalidad. } \\
\text { - Enumera las diferentes actividades que } \\
\text { se van a realizar durante la sesión, espe- } \\
\text { cificando su orden y temporalización. } \\
\text { - Especifica cuáles de las dinámicas y acti- } \\
\text { vidades van a realizarse de manera indivi- } \\
\text { dual, cuáles en pareja y cuáles en grupo. }\end{array}$ \\
\hline $\begin{array}{l}\text { Plataforma, } \\
\text { herramientas } \\
\text { y apps }\end{array}$ & $\begin{array}{l}\text { Especifica cuál va a ser la plata- } \\
\text { forma en la que se va a llevar a } \\
\text { cabo la sesión en línea y antici- } \\
\text { pa posibles problemas técnicos } \\
\text { y de conexión. }\end{array}$ & $\begin{array}{l}\text { Contenidos } \\
\text { y material }\end{array}$ & $\begin{array}{l}\text { Elabora un listado del material y los recur- } \\
\text { sos que van a emplearse durante la sesión, } \\
\text { tanto analógicos como digitales. }\end{array}$ \\
\hline $\begin{array}{l}\text { Objetivo(s) } \\
\text { general(es) de } \\
\text { la sesión }\end{array}$ & $\begin{array}{l}\text { Detalla los objetivos didácticos } \\
\text { generales de la sesión, hacien- } \\
\text { do especial hincapié en aque- } \\
\text { llos que tienen que ver con el } \\
\text { desarrollo de la competencia } \\
\text { digital en los estudiantes. }\end{array}$ & Evaluación & $\begin{array}{l}\text { Indica cuáles van a ser los instrumentos de } \\
\text { (auto)evaluación y su propósito. }\end{array}$ \\
\hline
\end{tabular}

Plantilla diseñada para facilitar la implementacion del modelo TPACK.

Fuente: https://rb.gy/gkrbnw 
Figura 14. «Observación» del CDCO (apartado «Procedimiento de observación»)

CDCO Manual de usuario Diagnóstico del centro Diagnóstico CDD Planificación Acción Observación Reflexión Mis herramientas Ejemplo de caso +

\section{Procedimiento de observación}

Protocolo de observación en el aula

1. PREOBSERVACIÓN

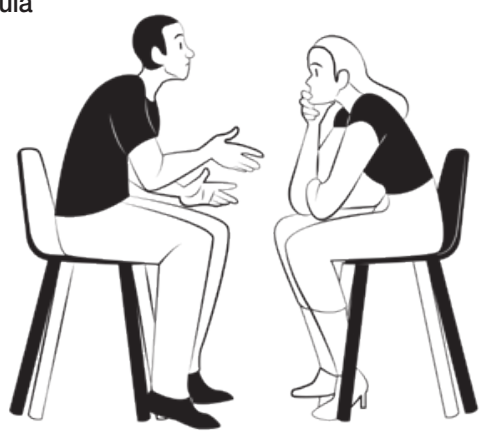

- Observador y docente pactan o consensúan las áreas y los criterios de evaluación con anterioridad a la sesión de observación.

- El docente da acceso a la programación del curso, a los materiales digitales y a su EVA al observador.

- Discusión entre ambos de objetivos de observación, categorías que se deben observar y otra información relevante para el proceso, como los contenidos, información previa sobre el alumnado o las dinámicas de clase.

\section{OBSERVACIÓN}

2.1. Online asíncrono $(\mathrm{OA})$

- La observación del EVA y de otros materiales en línea es conveniente no realizarla al inicio del curso, así el observador podrá ver muestras realizadas por los estudiantes, tener una idea de la participación del alumnado y de la interacción docente-discentes a lo largo del tiempo.

2.2. Online síncrono (OS)

- El observador graba la sesión en línea como muestra registrada del desempeño del docente.

- Al término de la sesión, la grabación se guardará en un lugar al que ningún agente externo tenga acceso, a excepción del docente y el observador.

- Recordad que se ha de seguir la normativa de derechos de imagen que rija en el centro a fin de operar dentro de la legislación que este aplique.

\section{POSOBSERVACIÓN}

- Reunión entre el observador y el docente con el propósito de que este último pueda responder y aclarar posibles dudas o cuestiones del observador. 
Figura 15. «Observación» del CDCO (apartado «Mi rúbrica de observación y evaluación»)

\section{Mi rúbrica de observación y evaluación}

Rúbrica de evaluación de criterios de observación y evaluación del proceso a fin de orientar al profesorado en cuanto a la selección y utilización de las tecnologías en los proyectos docentes (adaptado de TPACK).

\begin{tabular}{|c|c|c|c|c|}
\hline Criterios & Usuario experto & Usuario notable & Usuario medio & Usuario novato \\
\hline $\begin{array}{c}\text { Objetivos } \\
\text { curriculares y } \\
\text { tecnológicos } \\
\text { (Uso de la tecno- } \\
\text { logía basada en el } \\
\text { currículo) }\end{array}$ & $\begin{array}{l}\text { Las tecnologías se- } \\
\text { leccionadas en la } \\
\text { planificación didác- } \\
\text { tica están estrecha- } \\
\text { mente alineadas con } \\
\text { uno o más objetivos } \\
\text { curriculares. }\end{array}$ & $\begin{array}{l}\text { Las tecnologías se- } \\
\text { leccionadas en la } \\
\text { planificación didác- } \\
\text { tica están alineadas } \\
\text { con uno o más obje- } \\
\text { tivos curriculares. }\end{array}$ & $\begin{array}{l}\text { Las tecnologías se- } \\
\text { leccionadas en la } \\
\text { planificación didácti- } \\
\text { ca están parcialmen- } \\
\text { te alineadas con uno } \\
\text { o más objetivos cu- } \\
\text { rriculares. }\end{array}$ & $\begin{array}{l}\text { Las tecnologías se- } \\
\text { leccionadas en la } \\
\text { planificación didác- } \\
\text { tica no están alinea- } \\
\text { das con uno o más } \\
\text { objetivos curricu- } \\
\text { lares. }\end{array}$ \\
\hline $\begin{array}{c}\text { Estrategias de } \\
\text { enseñanza y } \\
\text { tecnologías } \\
\text { (Uso de la tecnolo- } \\
\text { gía en la enseñanza- } \\
\text { aprendizaje) }\end{array}$ & $\begin{array}{l}\text { El uso de la tecnolo- } \\
\text { gía apoya de manera } \\
\text { óptima las estrate- } \\
\text { gias de enseñanza. }\end{array}$ & $\begin{array}{l}\text { El uso de la tecnolo- } \\
\text { gía apoya las estra- } \\
\text { tegias de enseñanza. }\end{array}$ & $\begin{array}{l}\text { El uso de la tecno- } \\
\text { logía apoya mínima- } \\
\text { mente las estrategias } \\
\text { de enseñanza. }\end{array}$ & $\begin{array}{l}\text { El uso de la tecno- } \\
\text { logía no apoya las } \\
\text { estrategias de ense- } \\
\text { ñanza. }\end{array}$ \\
\hline $\begin{array}{l}\text { Selección de } \\
\text { tecnología } \\
\text { (Compatibilidad } \\
\text { con los objetivos } \\
\text { curriculares y las } \\
\text { estrategias de } \\
\text { enseñanza) }\end{array}$ & $\begin{array}{l}\text { La selección de } \\
\text { tecnología(s) es } \\
\text { ejemplar con res- } \\
\text { pecto a los objetivos } \\
\text { curriculares y las es- } \\
\text { trategias de ense- } \\
\text { ñanza. }\end{array}$ & $\begin{array}{l}\text { La selección de } \\
\text { tecnología(s) es } \\
\text { apropiada, aunque } \\
\text { no ejemplar, con res- } \\
\text { pecto a los objetivos } \\
\text { curriculares y las es- } \\
\text { trategias de ense- } \\
\text { ñanza. }\end{array}$ & $\begin{array}{l}\text { La selección de } \\
\text { tecnología(s) es poco } \\
\text { apropiada con res- } \\
\text { pecto a los objetivos } \\
\text { y las estrategias de } \\
\text { enseñanza. }\end{array}$ & $\begin{array}{l}\text { La selección de } \\
\text { tecnología(s) es ina- } \\
\text { propiada con res- } \\
\text { pecto a los objetivos } \\
\text { curriculares y las es- } \\
\text { trategias de ense- } \\
\text { ñanza. }\end{array}$ \\
\hline $\begin{array}{l}\text { Adecuación } \\
\text { (Contenido, } \\
\text { pedagogía y } \\
\text { tecnología en } \\
\text { forma conjunta) }\end{array}$ & $\begin{array}{l}\text { Contenido, estrate- } \\
\text { gias de enseñanza y } \\
\text { tecnología se articu- } \\
\text { lan completamen- } \\
\text { te entre sí dentro de } \\
\text { la planificación di- } \\
\text { dáctica. }\end{array}$ & $\begin{array}{l}\text { Contenido, estrate- } \\
\text { gias de enseñanza } \\
\text { y tecnologías se } \\
\text { articulan entre sí } \\
\text { dentro de la planifi- } \\
\text { cación didáctica. }\end{array}$ & $\begin{array}{l}\text { Contenido, estrate- } \\
\text { gias de enseñanza } \\
\text { y tecnología se ar- } \\
\text { ticulan parcialmen- } \\
\text { te entre sí dentro de } \\
\text { la planificación di- } \\
\text { dáctica. }\end{array}$ & $\begin{array}{l}\text { Currículo, estrate- } \\
\text { gias de enseñanza } \\
\text { y tecnología no se } \\
\text { articulan entre sí } \\
\text { dentro de la planifi- } \\
\text { cación didáctica. }\end{array}$ \\
\hline
\end{tabular}

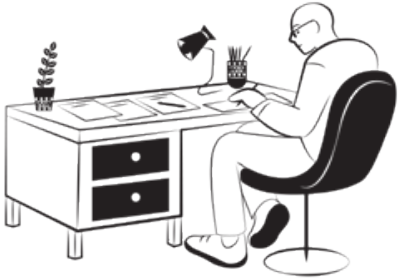

Fuente: https://rb.gy/gkrbnw 
Figura 16. "Observación» del CDCO (apartado «Propuesta de fichas para la observación»)

CDCO Manual de usuario Diagnóstico del centro Diagnóstico CDD Planificación Acción Observación Reflexión Mis herramientas Ejemplo de caso +

La primera vez que el docente y el observador se enfrentan a la elaboración de una ficha de observación pueden sentirse inseguros. Por ello, lo recomendable es que hablen, que consensúen los objetivos de la observación y los aspectos en los que les gustaría centrarse. De esa conversación puede salir la ficha que se usará para observar la grabación de la clase. A continuación, se sugieren dos propuestas de fichas de observación posibles para el segundo punto del área 3 del DigCompEdu (pedagogía digita):

\section{Área 3. Pedagogía digital:}

- Fomentar estrategias de enseñanza y aprendizaje eficientes, inclusivas e innovadoras.

- Facilitar la interacción docente-discentes.

- Favorecer la colaboración en línea.

- Desarrollar la autonomía en línea.

\section{FICHA 1}

Tipo de observación: holística.

Objetivo de la observación: la interacción docente-discente en la plataforma Zoom y capacidad para gestionar la plataforma para propiciar la interacción entre los alumnos.

1. Observa las diferentes interacciones y dinámicas de grupo que se dan en la clase: trabajo en parejas, grupo, individual, etc.

2. Haz una lista de las distintas dinámicas que se dan.

3. Anota la proporción aproximada de los diferentes tipos de interacción.

\begin{tabular}{|l|c|c|}
\hline \multicolumn{1}{|c|}{ Agrupamiento } & Tiempo & Dificultad de creación y gestión de salas de trabajo \\
\hline Clase abierta & $\%$ & \\
\hline Trabajo en parejas & $\%$ & \\
\hline Trabajo en grupos & $\%$ & \\
\hline Trabajo individual & $\%$ & \\
\hline Otros & $\%$ & \\
\hline
\end{tabular}

Ahora mira la grabación de la clase y completa la ficha. Para ello puedes parar la grabación las veces que consideres necesarias:

- Compara esos porcentajes con la percepción que tienes de lo que sucede en tus clases:

- ¿Coinciden?

- Si no coinciden, ¿te sorprenden las diferencias? ¿A qué crees que son debidas?

- Anota el grado de dificultad para la creación y gestión de las salas de trabajo.

- ¿Ha sido fácil crearlas?

- ¿Has podido «pasear» por las diferentes salas para monitorear a los alumnos?

- ¿Ha sido ágil la vuelta a la sala principal?

Conserva esa información para poder discutirla con tu observador.

Nota: en este artículo solo hemos incluido el ejemplo de la ficha 1. Para ver la ficha 2 remitimos al lector a la fuente de esta figura.

Fuente: https://rb.gy/gkrbnw 


\subsubsection{Reflexión}

La información aportada sobre la observación de las clases cobra valor cuando esta sirve como input, para el evaluado, de su actuación en el aula, del uso de los materiales analógicos y digitales y de su pericia en el manejo de las plataformas de enseñanza con el fin último del desarrollo de su CDD. Tras la grabación de la clase y una vez recogida la información sobre la observación en la herramienta previamente detallada, los datos recogidos han de analizarse debidamente, tanto por el evaluador como por el evaluado.

Analizar la información recogida significa relacionarla con los objetivos formativos del
La información aportada sobre la observación de las clases cobra valor cuando esta sirve como input, para el evaluado, de su actuación en el aula, del uso de los materiales analógicos y digitales y de su pericia en el manejo de las plataformas de enseñanza con el fin último del desarrollo de su CDD. [...] Analizar la información recogida significa relacionarla con los objetivos formativos del docente observado y con la observación llevada a cabo docente observado y con la observación llevada a cabo. De esa relación han de salir aspectos con los que observador y observado se sientan satisfechos, aspectos que se consideren mejorables y, probablemente, nuevos aspectos que observar derivados del proceso llevado a cabo basado en el modelo de investigación-acción. Observador y observado han de poder encontrar momentos en los que hablar de forma distendida sobre la percepción que cada uno extrae de la sesión de clase, contrastar esa percepción y argumentarla con la información recogida para darle un valor tan objetivo como sea posible. De ahí la importancia de la grabación de las clases, pues, si bien pueden existir aspectos en los que ambos no consigan ponerse de acuerdo, el hecho de poder volver a ver tantas veces como sea necesario los momentos que plantean conflicto y hablar sobre ellos permitirá poder seguir avanzando.

Además de los momentos de discusión sobre lo observado, es conveniente que el docente pueda reflexionar para poder extraer conclusiones sobre el grado de consecución de sus objetivos, de las dificultades que pueda sentir y del momento formativo en el que se encuentra. La observación, la discusión y la reflexión por sí mismas no garantizan el desarrollo de la CDD, sino que es necesario que el observado, tras ese proceso, sea capaz de diseñar un plan de acción para cubrir sus carencias formativas y seguir desarrollándose.

La importancia de este paso, crucial en el proceso, también queda reflejada en el CDCO, como se puede observar en el apartado «Reflexión» (véase figura 17).

La observación, la discusión y la
reflexión por sí mismas no garantizan
el desarrollo de la CDD, sino que es
necesario que el observado, tras ese
proceso, sea capaz de diseñar un plan
de acción para cubrir sus carencias
formativas y seguir desarrollándose 
Figura 17. «Reflexión» del CDCO

CDCO Manual de usuario Diagnóstico del centro Diagnóstico CDD Planificación Acción Observación Reflexión Mis herramientas Ejemplo de caso +

\section{Reflexión}

Análisis posterior a la observación realizada y reflexión sobre la acción, con el objeto de iniciar una nueva planificación y abrir un nuevo ciclo de investigación-acción.

Para facilitar el proceso de reflexión te proponemos que retomes los objetivos que te habías propuesto e intentes contestar a las siguientes cuestiones:

¿Los datos que me han aportado las fichas de observación y el diario de clases son suficientes? ¿Es necesario grabar alguna sesión más?

¿Estoy satisfecho con la experiencia llevada a cabo?

¿La observación de la clase grabada y los datos de las fichas de observación coinciden con mi percepción?

¿Qué información me aporta el diario y demás fichas de observación?

¿Los comentarios de mi observador coinciden con los datos que yo he extraído? ¿Hemos tenido oportunidad de discutir los aspectos en los que no coincidimos?

¿Los comentarios de mi observador son lo suficientemente claros para mí? ¿Necesito alguna aclaración?

¿He conseguido cubrir los objetivos que me había propuesto? ¿En qué grado puedo afirmar que se han conseguido?

¿Estoy preparado para iniciar otro ciclo formativo?

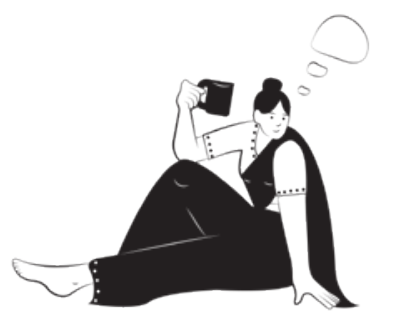

Fuente: https://rb.gy/gkrbnw

\subsubsection{Mis herramientas}

En este espacio los docentes tienen a su disposición más información desde una perspectiva visual y práctica que puede serles útil para el desarrollo de su competencia digital y para la integración de la tecnología en su actividad docente: «Matriz de integración de tecnología», "Cómo evaluar los recursos educativos digitales", "Competencias básicas según DigCompEdu", "Marco de competencia digital para ciudadanos", "Áreas de compentencia (MCCDD)", «EduPills: cápsulas formativas para el profesorado» y «Tabla periódica de apps y plataformas 
para profes». A continuación, se detalla cómo queda reflejada parte de esta información en el apartado correspondiente del CDCO (véanse figuras 18 a 22).

Figura 18. «Mis herramientas» del CDCO (apartado «Matriz de integración de tecnología»)

CDCO Manual de usuario Diagnóstico del centro Diagnóstico CDD Planificación Acción Observación Reflexión Mis herramientas Ejemplo de caso +

\section{Matriz de integración de tecnología}

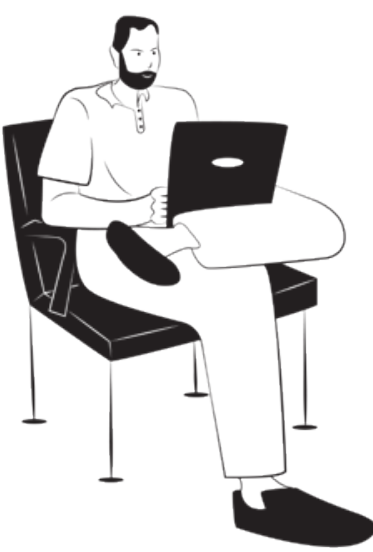

La matriz de integración de tecnología proporciona un marco de trabajo para describir y enfocarse en el uso de la tecnología para mejorar aprendizajes. La matriz, desarrollada por el Centro de Tecnología Educativa de Florida, incorpora cinco características interdependientes de los ambientes de aprendizaje significativos: activos, colaborativos, constructivos, auténticos y dirigidos a metas. Estas características están asociadas con cinco niveles de integración de tecnología: entrada, adopción, adaptación, infusión y transformación. Juntas, las cinco características de los entornos de aprendizaje significativos y los cinco niveles de integración tecnológica crean una matriz de 25 celdas, como se ilustra en la imagen.

\begin{tabular}{|c|c|c|c|c|c|}
\hline & ENTRADA & ADOPCIÓN & ADAPTACIÓN & INFUSIÓN & TRANSFORMACIÓN \\
\hline $\begin{array}{l}\begin{array}{r}\text { Niveles de } \\
\text { integración } \\
\text { de tecnología }\end{array} \\
\begin{array}{l}\text { Características } \\
\text { de los ambientes } \\
\text { de aprendizaje }\end{array}\end{array}$ & $\begin{array}{l}\text { El maestro co- } \\
\text { mienza a usar } \\
\text { tecnologías para } \\
\text { presentar conte- } \\
\text { nidos a los estu- } \\
\text { diantes }\end{array}$ & $\begin{array}{l}\text { El maestro dirige } \\
\text { a los alumnos en } \\
\text { el uso conven- } \\
\text { cional y de pro- } \\
\text { cedimiento de las } \\
\text { herramientas }\end{array}$ & $\begin{array}{l}\text { El maestro facilita } \\
\text { a los alumnos la } \\
\text { exploración y el } \\
\text { uso independien- } \\
\text { te de las herra- } \\
\text { mientas }\end{array}$ & $\begin{array}{l}\text { El maestro pro- } \\
\text { vee el contexto } \\
\text { de aprendizaje y } \\
\text { los estudiantes } \\
\text { escogen las he- } \\
\text { rramientas para } \\
\text { lograr el resul- } \\
\text { tado }\end{array}$ & $\begin{array}{l}\text { El maestro alienta el } \\
\text { uso innovador de las } \\
\text { herramientas, que } \\
\text { se usan para faci- } \\
\text { litar actividades de } \\
\text { aprendizaje de alto } \\
\text { nivel que no serían } \\
\text { posibles sin la tec- } \\
\text { nología }\end{array}$ \\
\hline ACTIVO & $\begin{array}{l}\text { ENTRADA ACTIVA } \\
\text { La información es }\end{array}$ & $\begin{array}{l}\text { ADOPCIÓN } \\
\text { ACTIVA }\end{array}$ & $\begin{array}{l}\text { ADAPTACIÓN } \\
\text { ACTIVA }\end{array}$ & $\begin{array}{l}\text { INFUSIÓN } \\
\text { ACTIVA }\end{array}$ & $\begin{array}{l}\text { TRANSFORMACIÓN } \\
\text { ACTIVA }\end{array}$ \\
\hline $\begin{array}{l}\text { Los estudiantes se in- } \\
\text { volucran activamente } \\
\text { en el uso de la tecno- } \\
\text { logía en vez de solo } \\
\text { recibir información } \\
\text { pasivamente de ella }\end{array}$ & $\begin{array}{l}\text { recibida pasiva- } \\
\text { mente }\end{array}$ & $\begin{array}{l}\text { Uso convencional } \\
\text { y procesal de las } \\
\text { herramientas }\end{array}$ & $\begin{array}{l}\text { Uso convencional } \\
\text { independiente } \\
\text { de herramientas, } \\
\text { algo de elección y } \\
\text { exploración }\end{array}$ & $\begin{array}{l}\text { Elección y uso } \\
\text { regular y auto- } \\
\text { dirigido de las } \\
\text { herramientas }\end{array}$ & $\begin{array}{l}\text { Uso extenso y poco } \\
\text { convencional de las } \\
\text { herramientas }\end{array}$ \\
\hline
\end{tabular}




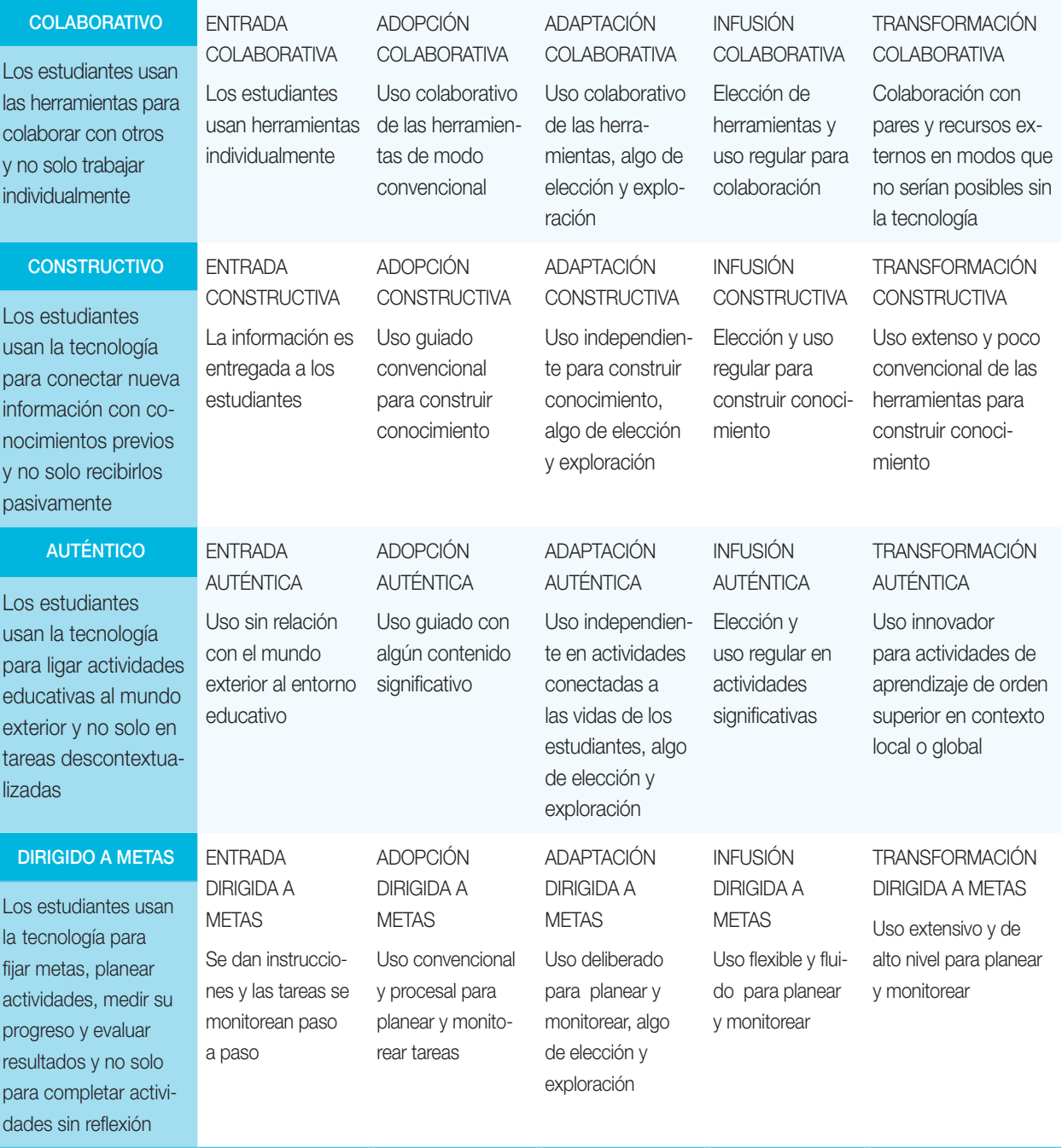

La matriz de integración de tecnología fue desarrollada por el Centro de Tecnología Educativa de Florida en la Facultad de Educación de la Universidad de South Florida. Para obtener más información, vídeos de ejemplos y recursos de desarrollo profesional relacionados, visite http://mytechmatrix.org. Esta página puede ser reproducida por las escuelas y los distritos para el desarrollo profesional y la instrucción previa al servicio. Todo otro uso requiere permiso por escrito del FCIT. @ 2005-2017 University of South Florida.

Traducción al español (no oficial): http://www.eduteka.org/articulos/tim

Fuente: https://rb.gy/gkrbnw 
Figura 19. «Mis herramientas» del CDCO (apartado «EduPills: cápsulas formativas para el profesorado»)

CDCO Manual de usuario Diagnóstico del centro Diagnóstico CDD Planificación Acción Observación Reflexión Mis herramientas Ejemplo de caso +

\title{
Edupills: cápsulas formativas para el profesorado
}

¡Descarga ya la app en tu móvil!

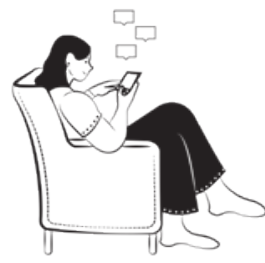

\begin{abstract}
La formación continua de los docentes y la mejora de sus competencias digitales son fundamentales para la transformación de la escuela. En este sentido, merece la pena fijarse en la aplicación EduPills, lanzada por el INTEF.
\end{abstract}

Fáciles de utilizar y siempre disponibles, EduPills proporciona breves cápsulas formativas pensadas para docentes. Estos minicursos gratuitos ponen a disposición de los profesores «nuevos conceptos relacionados con su trabajo», basándose en las áreas del MCCDD.

Una apuesta por el mobile learning y por la mejora de habilidades y destrezas digitales que se irá ampliando periódicamente con nuevos contenidos.

Si eres profesor, jdescárgate la app en tu móvil y sigue aprendiendo cada día!

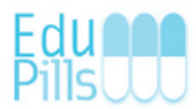

Fuente: https://rb.gy/gkrbnw

Figura 20. «Mis herramientas» del CDCO (apartado «Marco de Competencia Digital para Ciudadanos»)

CDCO Manual de usuario Diagnóstico del centro Diagnóstico CDD Planificación Acción Observación Reflexión Mis herramientas Ejemplo de caso ++

\section{Aprendiendo a nadar en el océano digital}

Marco de Competencia Digital para Ciudadanos

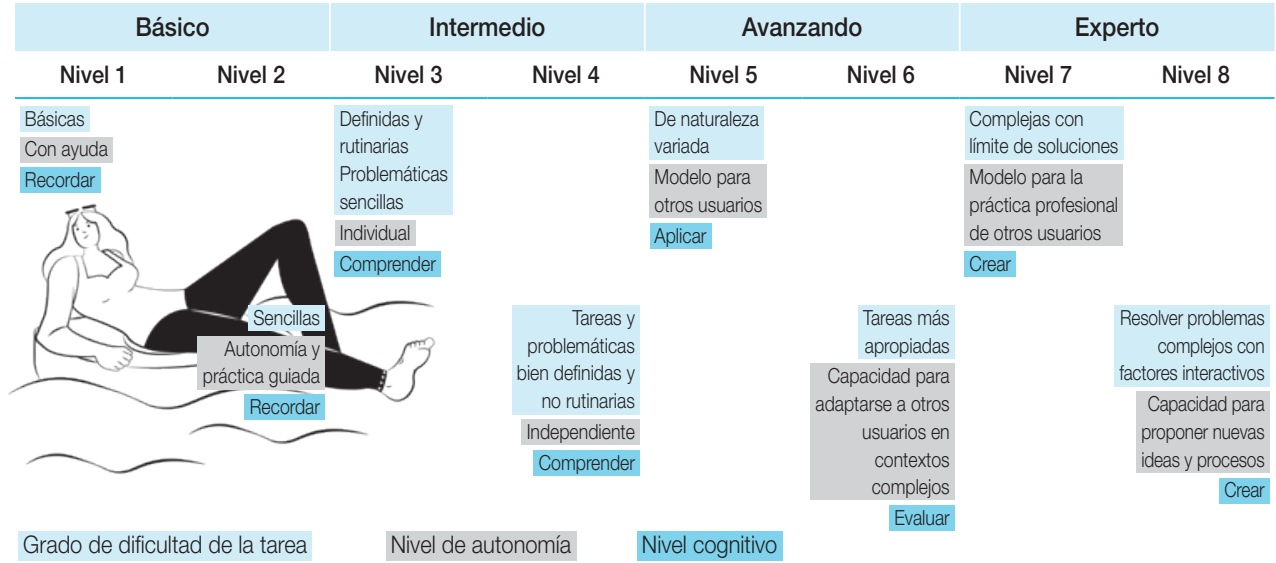

Fuente: https://ec.europa.eu/jrc/en/science-update/new-digcomp-report-develops-proficiency-levels

Fuente: https://rb.gy/gkrbnw 
Figura 21. «Mis herramientas» del CDCO (apartado «Cómo evaluar los recursos educativos digitales»)

CDCO Manual de usuario Diagnóstico del centro Diagnóstico CDD Planificación Acción Observación Reflexión Mis herramientas Ejemplo de caso + +

\section{Cómo evaluar los recursos educativos digitales}

A la hora de seleccionar las herramientas y recursos educativos digitales es de suma importancia que se valore su usabilidad, es decir, su capacidad para hacer más productivas las tareas. La tabla de "Criterios de evaluación de recursos educativos digitales» de Nokelainen (2005) actúa como una herramienta informática de evaluación de usabilidad.

La tabla incluye 10 dimensiones que hacen operativas en 56 subdimensiones. Estas 10 dimensiones son: 1. Control del alumno; 2. Actividad del alumno; 3. Aprendizaje cooperativo/colaborativo; 4. Orientación objetivos; 5. Aplicabilidad; 6. Valor añadido; 7. Motivación; 8. Valoración del conocimiento previo; 9. Flexibilidad; 10. Feedback.

\begin{tabular}{|c|c|}
\hline Criterios & Subdimensiones \\
\hline 1. Learner control & $\begin{array}{l}\text { Minimum memory load; meaningful encoding; responsibility for learning; user control; } \\
\text { elaboration. }\end{array}$ \\
\hline 2. Learner activity & $\begin{array}{l}\text { Reflective thinking; problem-based learning; use of primary data sources; immersion; } \\
\text { ownership; primary data source (for PBL) (for teacher); facilitative teacher (for teacher); didactic } \\
\text { teacher (for teacher); individual/distance learning (for teacher). }\end{array}$ \\
\hline $\begin{array}{l}\text { 3. Cooperative/ } \\
\text { Collaborative } \\
\text { learning }\end{array}$ & $\begin{array}{l}\text { Support for conversation and dialogue; group work; asynchronous social navigation; } \\
\text { synchronous social navigation; asynchronous social navigation monitoring (for teacher); } \\
\text { synchronous social navigation monitoring (for teacher); tertiary courseware. }\end{array}$ \\
\hline 4. Goal orientation & $\begin{array}{l}\text { Explicit goals; usefulness of goals; focus on results; focused goals; monitor one's own studies } \\
\text { (pedagogic feedback); set one's own goals (for teacher). }\end{array}$ \\
\hline 5. Applicability & $\begin{array}{l}\text { Authentic material; perceived usefulness; learning by doing; adequate material for the learners } \\
\text { needs (human development); pretesting and diagnostics; prompting; fading: scaffolding; } \\
\text { meaningful encoding. }\end{array}$ \\
\hline 6. Added value & $\begin{array}{l}\text { Overall added value for learning; effectiveness for learning; added value of pictures; added } \\
\text { value of sounds; added value of animations. }\end{array}$ \\
\hline 7. Motivation & Intrinsic goal orientation; extrinsic goal orientation; meaningfulness of studies; immersion. \\
\hline $\begin{array}{l}\text { 8. Valuation } \\
\text { of previous } \\
\text { knowledge }\end{array}$ & Prerequisites; elaboration; examples. \\
\hline 9. Flexibility & Pretesting and diagnostics; task decomposition; repetitive tasks. \\
\hline 10. Feedback & Encouraging feedback; accurate feedback; errorless learning. \\
\hline 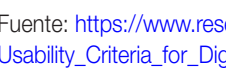 & $\begin{array}{l}\text { chgate.net/publication/235329276_The_Technical_and_Pedagogical_ } \\
\text { _Learning_Material }\end{array}$ \\
\hline
\end{tabular}

Fuente: https://rb.gy/gkrbnw 
Figura 22. «Mis herramientas» del CDCO (apartado «Tabla periódica de apps y plataformas para profes»)

CDCO Manual de usuario Diagnóstico del centro Diagnóstico CDD Planificación Acción Observación Reflexión Mis herramientas Ejemplo de caso ++

\section{Tabla periódica de apps \\ y plataformas para profes}

Tabla periódica de

Descárgala + Comparte otras plataformas

\section{Apps y plataformas para profesores}

\section{(c)}
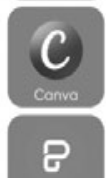

8
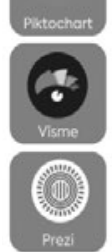

(ำำ
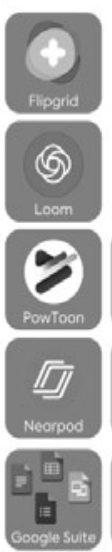

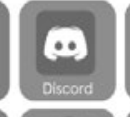

Creación de contenido

Comunicación y comunidad

Evaluación del aprendizaje
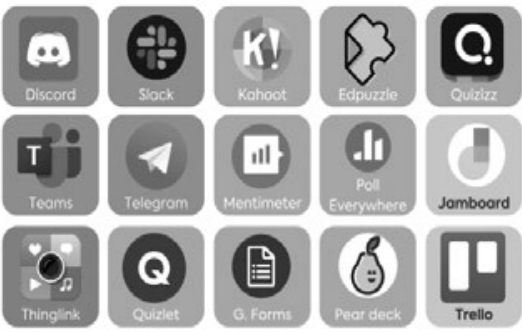

Organización de ideas y pizarrones

Gestión de

contenido

Contenido educativo

por@andreaoviedov

우 in $y$ @andreaoviedov
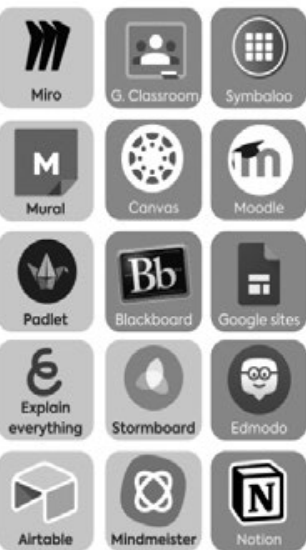
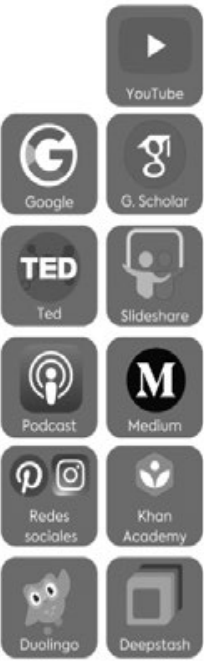

Khan
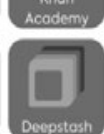

Fuente: https://view.genial.ly/600caa6d56e45e74763a41f4/horizontal-infographic-review-aplicaciones-para-profesores.

\subsubsection{Ejemplo de caso}

Para facilitar la tarea al docente, se ha incluido una sección en el CDCO en la que se ejemplifican cada uno de los pasos propuestos. De este modo se espera que observadores y docentes sin experiencia previa en diseños de planes de formación puedan familiarizarse con los procesos que se llevan a cabo y con las
Con la finalidad de facilitar la tarea al docente, en el CDCO se ha incluido la sección «Ejemplo de caso», donde observadores y profesorado sin experiencia previa en diseños de planes de formación pueden familiarizarse con los procesos 
herramientas que se utilizan. El ejemplo que aquí incluimos parte de la siguiente simulación: una profesora quiere introducir aplicaciones digitales en sus clases con el objetivo de incrementar la participación e implicación de sus alumnos en las sesiones que imparte. El plan de formación que se usa de ejemplo se inicia con el diagnóstico de la CDD. Se incluyen los planes de trabajo y las fichas que se utilizarían para lograr ese objetivo (véanse figuras 23 y 24).

\author{
El ejemplo de caso que \\ aquí incluimos parte de una \\ profesora que quiere introducir \\ aplicaciones digitales en las clases \\ que imparte con el objetivo de \\ incrementar la participación e \\ implicación de su alumnado
}

Figura 23. «Ejemplo de caso» del CDCO (apartado «Presentación del caso»)

CDCO Manual de usuario Diagnóstico del centro Diagnóstico CDD Planificación Acción Observación Reflexión Mis herramientas Ejemplo de caso ++

\section{Ejemplo de caso}

\section{Presentación del caso}

Emily Brönte es profesora de lengua y literatura de bachillerato y recientemente se ha propuesto introducir nuevas herramientas digitales en sus clases para mejorar los niveles de participación y motivación de su alumnado. Para ello, Emily ha decidido ir introduciendo cada semana una nueva aplicación, recoger información de cómo le ha funcionado, analizar esos datos, ver si los resultados son positivos en relación con la participación y la motivación de los alumnos y también con su experiencia como usuaria de la nueva aplicación. En las sucesivas páginas de este apartado resumimos el proceso que ha seguido Emily basándose en la información y en los pasos estipulados en los diferentes apartados del CDCO que recientemente han recibido en su centro.

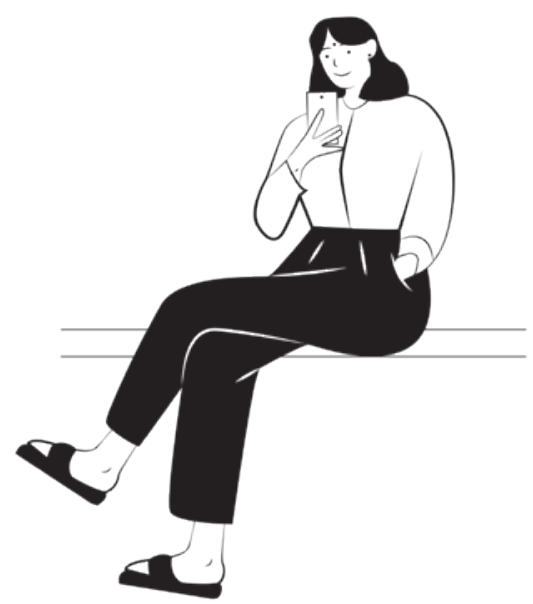

Nota: en este artículo no hemos reproducido todas las páginas del ejemplo de caso. El lector interesado podrá encontrarlas en la fuente de la figura. 
Figura 24. «Ejemplo de caso» del CDCO (apartado «Fase de autodiagnóstico»)

CDCO Manual de usuario Diagnóstico del centro Diagnóstico CDD Planificación Acción Observación Reflexión Mis herramientas Ejemplo de caso ++

\section{FASE de aUtodiagnóstico}

\section{Diagnóstico CDD}

Lo primero que hizo Emily Brönte fue leer las instrucciones del «Manual de Usuario» del CDCO y realizar el test diagnóstico de su centro. Seguidamente, llevó a cabo el test DigCompEdu de CDD para ver qué puntuación obtenía. La verdad es que no estaba mal del todo. Este es el resultado general obtenido (puedes descargar el informe completo a continuación):

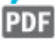

EUSurvey-

Survey

\section{Si su puntuación está entre 34 y 49, es «Integrador» (B1)}

Esto significa: experimenta con tecnologías digitales en una variedad de contextos y para diversos propósitos, integrándolos en muchas de sus prácticas docentes. Los usa creativamente para mejorar diversos aspectos de su compromiso profesional. Está impaciente por ampliar su repertorio de prácticas. Se beneficiará al aumentar la comprensión sobre qué herramientas funcionan mejor en qué situaciones y sobre cómo adaptar las tecnologías digitales a las estrategias y métodos pedagógicos. Trate de darse más tiempo para la reflexión y la adaptación, complementado con el intercambio de estímulos colaborativos y de conocimientos, para llegar al siguiente paso, "Experto» (B2).

De los 6 niveles posibles había quedado hacia la mitad, no todo estaba perdido. Con esa información ya podía plantearse un plan de acción.

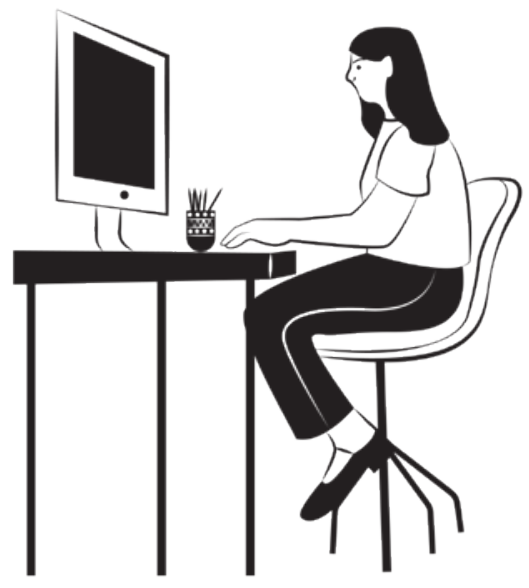

Fuente: https://rb.gy/gkrbnw 


\section{Evaluación del diseño del CDCO}

En el estado en el que se encuentra la propuesta solo se han podido pilotar algunas secciones del CDCO en contextos de enseñanza no reglada y en niveles universitarios; en concreto, aquellas que hacen referencia al docente, a la planificación y a su plan de observación.

En relación con las secciones pilotadas, puede afirmarse que el nivel de satisfacción es alto debido a los siguientes aspectos: facilidad de uso y acceso a las diferentes secciones, buena secuenciación de la información en el cuaderno y en las páginas incorporadas, enlaces de interés, información visualmente atractiva y actualizada, fácil comprensión de la información que ofrece, instrucciones claras, fichas adaptables y customizables, cuestionarios fácilmente administrables y naturaleza colaborativa y personalizable.

Por otro lado, durante el proceso de pilotaje se ha podido apreciar una clara inclinación por parte de los agentes implicados en la aplicación de la propuesta de observación a añadir, duplicar y modificar contenido de las diferentes secciones, especialmente en relación con las fichas de observación. En este sentido, la selección de la herramienta OneNote parece haber sido la correcta, pues su naturaleza versátil y fácilmente editable permite la personalización y adaptación del contenido a las necesidades de los diferentes agentes implicados en el proceso. No obstante, con el propósito de comprobar el grado de utilidad del CDCO como herramienta clave en los procesos de formación y transformación digital de los centros, sería necesario aplicar la propuesta en un mayor número de centros de enseñanza a fin de obtener datos cuantitativos y feedback cualitativo de su efectividad y poder seguir mejorando los aspectos que así lo requieran.

\section{Conclusiones e implicaciones futuras}

Para alcanzar el objetivo principal propuesto, esto es, el desarrollo de la CDD en contexto y facilitar así la transición hacia la digitalización de los procesos de enseñanza, aprendizaje y evaluación, se ha propuesto y diseñado un CDCO en OneNote, el cual, según se ha podido corroborar, sirve a tal propósito gracias a tres factores:

- Se trata de una herramienta fácilmente accesible y flexible en su administración que potencia el trabajo cooperativo entre los agentes implicados.

- Ofrece información suficiente para llevar a cabo el plan de formación de centro y el plan de formación individual, sin limitación de espacio o tiempo.

- Proporciona pautas y herramientas que garantizan el acompañamiento al docente a lo largo del proceso. 
Como se ha señalado anteriormente, es necesario difundir la propuesta a un número mayor de centros de enseñanza de diferentes niveles para poder hacer los ajustes necesarios y valorar su efectividad. Por otro lado, la presente línea de trabajo, centrada en el desarrollo de la CDD, puede ampliarse para dar cabida a otras cuestiones relacionadas con la formación continua del profesorado, propiciando así que los docentes se conviertan en expertos capaces de crear, diseñar y gestionar su propio entorno personal de aprendizaje (EPA) y guiar a sus estudiantes en la gestión del suyo, una visión muy en línea con las propuestas de autores como Castañeda et al. (2018).

Para terminar, con el fin de conferir una mayor flexibilidad al plan de formación y observación propuesto y facilitar su adopción e implementación por el mayor número posible de instituciones y comunidades educativas, se sugiere la posibilidad de emplear otras herramientas digitales alternativas, pero con prestaciones similares a OneNote (colaborativas, de fácil acceso y de uso flexible), para acometer el diseño del cuaderno colaborativo.

\section{Referencias bibliográficas}

Ala-Mutka, K. (2011). Mapping Digital Competence: Towards a Conceptual Understanding. JRC-IPTS.

Carr, W. y Kemmis, S. (1986). Becoming Critical: Education Knowledge and Action Research (1. ${ }^{a}$ ed.). Routledge. https://doi.org/10.4324/ 9780203496626

Carretero, S., Vuorikari, R. y Punie, Y. (2017). DigComp 2.1: The Digital Competence Framework for Citi-Zens with Eight Proficiency Levels and Examples of Use. Publications Office of the European Union. https://bit. ly/2pGtGll

Castañeda, L., Esteve, F. y Adell, J. (2018). ¿Por qué es necesario repensar la competencia docente para el mundo digital? Revista de Educación a Distancia, 56, 1-20. https://doi. org/10.6018/red/56/6

Comisión Europea. (2017). Evaluar la competencia digital docente. https://ec.europa.eu/

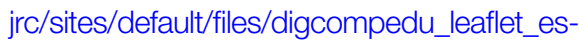
nov2017pdf.pdf

Comisión Europea. (2021). Plan de acción de educación digital 2021-2027: adaptar la educación y la formación a la era digital. https:// ec.europa.eu/education/education-inthe-eu/digital-education-action-plan_en

Delgado, P. (2020). La capacitación en línea, el gran reto de la educación en línea. Observatorio de innovación educativa. Tecnológico de Monterrey. https://observatorio.tec.mx/edunews/capacitacion-docente-covid

DiCTE. (Ed.). (2017). About DiCTE Project. Developing ICT in Teacher Education. https://bit. ly/2TkTVlp

Edkey, M. T. y Roehrich, H. (2013). A faculty observation model for online instructors: observing faculty members in the online classroom. Online Journal of Distance Learning Administration, 16(2). http://www.westga.edu/ distance/ojdla/summer162/eskey_roehrich 162.html

Ferrari, A. (2012). Digital Competence in Practice: An Analysis of Frameworks. European Commission; Joint Research Centre; Institute for Prospective Technological Studies. https://doi.org/10.2791/82116

Ferrari, A. (2013). Gallaudet University. https:// my.gallaudet.edu/online-teaching-certificate-information/repository/important/ guidelines-for-observation-of-online-teaching 
Ferrari, A. (autor), Punie, Y. y Brečko, B. N. (Eds.). (2013). DigComp: A Framework for Developing and Understanding Digital Competence in Europe. European Commission; Joint Research Centre; Institute for Prospective Technological Studies. https://doi. org/10.2788/52966

Gudmundsdottir, S. y Shulman, L. (1987). Pedagogical content Knowledge in social studies. Scandinavian Journal of Educational Research, 31(2), 59-70, https://doi. org/10.1080/0031383870310201

INTEF. (2017). Marco Común de Competencia Digital Docente. http://aprende.intef.es/sites/ default/files/2018-05/2017_1020_MarcoCom\%C3\%BAn-de-Competencia-DigitalDocente.pdf

INTEF. (2019). Resumen Informe Horizon. https:// issuu.com/etwinning/docs/2019_07_resu men_horizon_universidad_2019_intef_1_

Koehler, M. J. y Mishra, P. (2009). What is technological pedagogical content knowledge? Contemporary Issues in Technology and Teacher Education, 9(1), 60-70.

Martin, A. y Grudziecki, J. (2006). DigEuLit: concepts and tools for digital literacy development. Innovation in Teaching and Learning in Information and Computer Sciences, 5(4), 249-267. https://doi.org/10.11120/ital.2006. 05040249

Ministerio de Educación y Formación Profesional. (2020). Resolución de 2 de julio de 2020, de la Dirección General de Evaluación y Cooperación Territorial, por la que se publica el Acuerdo de la Conferencia Sectorial de Educación sobre el Marco de Referencia de la Competencia Digital Docente (Boletín Oficial del Estado [BOE] núm. 191, de 13 de julio de 2020).

Montmany, B. y González, M. V. (2019). La observación de la práctica docente: una conjunción de miradas. En J. T. Pujolà y C. López Ferrero (Eds.), La didáctica de lenguas de par en par: diálogo entre teoría y práctica (pp. 284-301). Difusión.

Nokelainen, P. (2005). The technical and pedagogical usability criteria for digital learning material. En P. Kommers y G. Richards (Eds.), Proceedings of ED-MEDIA 2005World Conference on Educational Multimedia, Hypermedia \& Telecommunications (pp. 1.011-1.016). Montreal, Canadá: Association for the Advancement of Computing in Education (AACE). https://www.learntechlib. org/primary/p/20212

ProFuturo. (2019). Marco Europeo para la Competencia Digital Docente. DigCompEdu. https:// profuturo.education/topics/un-marco-europeopara-la-competencia-digital-de-los-educado res-digcompedu/

Richards, J. C. y Lockhart, C. (1998). Estrategias de reflexión sobre la enseñanza de idiomas. Cambridge University Press.

Tobin, T. J., Mandernach, B. J. y Taylor, A. H. (2015). Evaluating Online Teaching: Implementing Best Practices. John Wiley \& Sons.

UNESCO. (2020). https://infogram.com/finalunesco-education-covid-19-data-1hke60 d1 $1 \times 7 m 525 r$

UNESCO Institute for Statistics (UIS). (2020). Encuesta sobre las respuestas de la educación nacional al cierre de escuelas COVID-19. Primera iteración. http://tcg.uis.unesco.org/ survey-education-covid-school-closures/

UNICEF. (2020). La falta de igualdad en el acceso a la educación a distancia en el contexto de la COVID-19 podría agravar la crisis mundial del aprendizaje. https://www.unicef. org/es/comunicados-prensa/falta-igualdadacceso-educacion-distancia-podria-agravar-crisis-aprendizaje

Vallejo, C. (2013). Monográfico: Introducción de las tecnologías en laeducación TPACK. Cajón de Sastre. http://recursostic.educacion.es/ observatorio/web/es/component/content/ article/1092-monografico-introduccion-delas-tecnologias-en-la-educacion?start=1 